\title{
MAXIMAL REGULAR BOUNDARY VALUE PROBLEMS IN BANACH-VALUED FUNCTION SPACES AND APPLICATIONS
}

\author{
VELI B. SHAKHMUROV
}

Received 27 December 2004; Accepted 30 September 2005

The nonlocal boundary value problems for differential operator equations of second order with dependent coefficients are studied. The principal parts of the differential operators generated by these problems are non-selfadjoint. Several conditions for the maximal regularity and the Fredholmness in Banach-valued $L_{p}$-spaces of these problems are given. By using these results, the maximal regularity of parabolic nonlocal initial boundary value problems is shown. In applications, the nonlocal boundary value problems for quasi elliptic partial differential equations, nonlocal initial boundary value problems for parabolic equations, and their systems on cylindrical domain are studied.

Copyright (c) 2006 Hindawi Publishing Corporation. All rights reserved.

\section{Introduction, notations, and background}

Boundary value problems (BVPs) for differential operator equations (DOE) in $H$-valued (Hilbert space-valued) function spaces have been studied extensively by many researchers (see $[4-7,12,15,16,18,20,22,28-33,37-39]$ and the references therein). In these works Hilbert-valued function spaces essentially were considered. The main objective of the present paper is to discuss the nonlocal BVP for DOE with variable coefficients in Banach-valued function spaces. In this work, (1) at first, nonhomogenous BVP for ordinary DOE is considered; (2) partial DOE with dependent coefficients in principal part is considered; (3) boundary conditions are, generally, nonlocal; (4) operators containing equations and boundary conditions are, in general, unbounded; (5) nonlocal initial boundary value problems (IBVP) for parabolic DOE are considered. The maximal regularity, positivity and, Fredholmness of these problems in Banach-valued $L_{p}$-spaces are proved. These results are also applied to the nonlocal BVP for quasi elliptic partial differential equations, infinite systems of nonlocal BVP for elliptic equations with variable coefficients, and INBVP for parabolic equations on cylindrical domains.

Let $E$ be a Banach space. $L_{p}(\Omega ; E)$ denotes a space all of strongly measurable $E$-valued functions that are defined on a domain $\Omega \subset R^{n}$ with the norm

$$
\|f\|_{L_{p}}=\|f\|_{L_{p}(\Omega ; E)}=\left(\int\|f(x)\|_{E}^{p} d x\right)^{1 / p}, \quad 1 \leq p<\infty .
$$

Hindawi Publishing Corporation

International Journal of Mathematics and Mathematical Sciences

Volume 2006, Article ID 92134, Pages 1-26

DOI 10.1155/IJMMS/2006/92134 
By $L_{p, q}(\Omega)$ and $W_{p, q}^{l}(\Omega)$, we will denote a scalar-valued $(p, q)$-integrable function space and Sobolev space with mixed norms, respectively [8]. Let $B_{p q}^{s}$ denote the Besov space (see, e.g., [35, Section 2.3]).

A Banach space $E$ is said to be $\zeta$-convex space (see $[9-11,15,23])$ if there exists on $E \times E$ a symmetric real-valued function $\zeta(u, v)$ which is convex with respect to each of the variables, and satisfies the conditions

$$
\zeta(0,0)>0, \quad \zeta(u, v) \leq\|u+v\|, \quad \text { for }\|u\|=\|v\|=1 .
$$

In literature the $\zeta$-convex Banach spaces $E$ are often called UMD-spaces and written as $E \in \mathrm{UMD}$. It is shown in [10] that the Hilbert operator

$$
(H f)(x)=\lim _{\varepsilon \rightarrow 0} \int_{|x-y|>\varepsilon} \frac{f(y)}{x-y} d y
$$

is bounded in $L_{p}(R ; E), p \in(1, \infty)$, for those and only those spaces $E$ which possess the property of UMD spaces. UMD spaces include, for example, $L_{p}, l_{p}$ spaces and Lorentz spaces $L_{p q}, p, q \in(1, \infty)$.

Let $\mathbb{C}$ be a set of complex numbers. $S_{\varphi}$ denotes an open sector with vertex 0 , opening angle $2 \varphi$, which is symmetric with respect to the positive half-axis $\mathbb{R}_{+}$, that is,

$$
K_{\varphi}=\{\xi ; \xi \in \mathbb{C},|\arg \xi-\pi| \leq \pi-\varphi\}, \quad 0<\varphi \leq \pi .
$$

Let

$$
S_{\varphi}=\{\xi ; \xi \in \mathbb{C},|\arg \xi| \leq \pi-\varphi\}, \quad 0<\varphi \leq \pi .
$$

A linear operator $A$ is said to be positive in a Banach space $E$, with bound $M$ if $D(A)$ is dense on $E$ and

$$
\left\|(A+\xi I)^{-1}\right\|_{L(E)} \leq M(1+|\xi|)^{-1}
$$

with $\xi \in K_{\varphi}, \varphi \in(0, \pi]$, where $M$ is a positive constant and $I$ an identity operator in $E$, where $L(E)$ is a space of bounded linear operators acting in $E$. Sometimes instead of $A+\xi I$, will be written $A+\xi$ and denoted by $A_{\xi}$. The operator $A(t)$ is said to be positive in a Banach space $E$ uniformly with respect to $t$, if $D(A(t))$ is independent of $t, D(A(t))$ is dense in $E$, and

$$
\left\|(A(t)+\lambda I)^{-1}\right\| \leq \frac{M}{1+|\lambda|}
$$

for all $\lambda \in K(\varphi), \varphi \in(0, \pi]$.

It is known [35, Section 1.15.1] that there exist fractional powers $A^{\theta}$ of the positive operator $A$. Let $E\left(A^{\theta}\right)$ denote the space $D\left(A^{\theta}\right)$ with graphical norm defined as

$$
\|u\|_{E\left(A^{\theta}\right)}=\left(\|u\|^{p}+\left\|A^{\theta} u\right\|^{p}\right)^{1 / p}, \quad 1 \leq p<\infty,-\infty<\theta<\infty .
$$

Let $E_{1}$ and $E_{2}$ be two Banach spaces. By $\left(E_{1}, E_{2}\right)_{\theta, p}, 0<\theta<1,1 \leq p \leq \infty$, will be denoted an interpolation space for $\left\{E_{1}, E_{2}\right\}$ by the $K$-method [35, Section 1.3.1]. By $C(\Omega ; E)$ 
and $C^{(m)}(\Omega ; E)$ will be denoted spaces of $E$-valued bounded continuous and $m$-times continuously differentiable function on $\Omega$, respectively. Let $S\left(\mathbb{R}^{n} ; E\right)$ denote a Schwarz class, that is, the space of all $E$-valued rapidly decreasing smooth functions $\varphi$ on $\mathbb{R}^{n}$. The function $\Psi \in C\left(\mathbb{R}^{n} ; L\left(E_{1}, E_{2}\right)\right)$ is called a multiplier from $L_{p}\left(\mathbb{R}^{n} ; E_{1}\right)$ to $L_{q}\left(\mathbb{R}^{n} ; E_{2}\right)$ if the map $u \rightarrow K u=F^{-1} \Psi(\xi) F u, u \in S\left(\mathbb{R}^{n} ; E_{1}\right)$, is well defined and extends to a bounded linear operator

$$
K: L_{p}\left(\mathbb{R}^{n} ; E_{1}\right) \longrightarrow L_{q}\left(\mathbb{R}^{n} ; E_{2}\right)
$$

We denote the set of all multipliers from $L_{p}\left(\mathbb{R}^{n} ; E_{1}\right)$ to $L_{q}\left(\mathbb{R}^{n} ; E_{2}\right)$ by $M_{p}^{q}\left(E_{1}, E_{2}\right)$. For $E_{1}=$ $E_{2}=E$ we denote $M_{p}^{q}\left(E_{1}, E_{2}\right)$ by $M_{p}^{q}(E)$. Let

$$
H_{k}=\left\{\Psi_{h} \in M_{p}^{q}\left(E_{1}, E_{2}\right), h=\left(h_{1}, h_{2}, \ldots, h_{L}\right) \in Q\right\}
$$

be a collection of multipliers in $M_{p}^{q}\left(E_{1}, E_{2}\right)$. We say that $\Phi_{h}$ is a uniformly bounded multiplier with respect to $h$ if there exists a constant $C>0$, independent on $h \in B(h)$, such that

$$
\left\|F^{-1} \Psi_{h} F u\right\|_{L_{q}\left(\mathbb{R}^{n}, E_{2}\right)} \leq C\|u\|_{L_{p}\left(\mathbb{R}^{n}, E_{1}\right)}
$$

for all $h \in K$ and $u \in S\left(\mathbb{R}^{n} ; E_{1}\right)$.

The exposition of the theory of $L_{p}$-multipliers of the Fourier transformation, and some related references, can be found in [35, Sections 2.2.1-2.2.4]. On the other hand, in vector-valued function spaces, Fourier multipliers have been studied by $[11-13,18,26$, 27, 36].

A set $K \subset B\left(E_{1}, E_{2}\right)$ is called $\mathbb{R}$-bounded (see, e.g., $[9,11,12,18,36]$ ) if there is a constant $C$ such that for all $T_{1}, T_{2}, \ldots, T_{m} \in K$ and $u_{1}, u_{2}, \ldots, u_{m} \in E_{1}, m \in \mathbb{N}$,

$$
\int_{0}^{1}\left\|\sum_{j=1}^{m} r_{j}(y) T_{j} u_{j}\right\|_{E_{2}} d y \leq C \int_{0}^{1}\left\|\sum_{j=1}^{m} r_{j}(y) u_{j}\right\|_{E_{1}} d y,
$$

where $\left\{r_{j}\right\}$ is a sequence of independent symmetric $\{-1,1\}$-valued random variables on $\{0,1\}$ and $\mathbb{N}$ denotes the set of natural numbers.

A set $K(h) \subset B\left(E_{1}, E_{2}\right)$ depending on parameters $h=\left(h_{1}, h_{2}, \ldots, h_{L}\right) \in B(h) \in \mathbb{R}^{L}$ is called uniformly $\mathbb{R}$-bounded with respect to $h$ if there is a constant $C$ such that for all $T_{1}(h), T_{2}(h), \ldots, T_{m}(h) \in K$ and $u_{1}, u_{2}, \ldots, u_{m} \in E_{1}, m \in N$,

$$
\int_{0}^{1}\left\|\sum_{j=1}^{m} r_{j}(y) T_{j}(h) u_{j}\right\|_{E_{2}} d y \leq C \int_{0}^{1}\left\|\sum_{j=1}^{m} r_{j}(y) u_{j}\right\|_{E_{1}} d y,
$$

where a positive constant $C$ is independent of the parameter $h$.

Let

$$
\begin{gathered}
V_{n}=\left\{\xi: \xi=\left(\xi_{1}, \xi_{2}, \ldots, \xi_{n}\right) \in \mathbb{R}^{n}, \xi_{j} \neq 0\right\}, \\
U_{n}=\left\{\beta=\left(\beta_{1}, \beta_{2}, \ldots, \beta_{n}\right),|\beta| \leq n\right\}, \quad \xi^{\beta}=\xi_{1}^{\beta_{1}}, \xi_{2}^{\beta_{2}}, \ldots, \xi_{n}^{\beta_{n}} .
\end{gathered}
$$


Definition 1.1. A Banach space $E$ is said to be the space satisfying a multiplier condition with respect to $p \in(1, \infty)$ when for $\Psi \in C^{(n)}\left(\mathbb{R}^{n} ; B(E)\right)$, if the sets

$$
\Psi(\xi):\left\{\xi^{\beta} D_{\xi}^{\beta} \Psi(\xi): \xi \in V_{n}, \beta \in U_{n}\right\}
$$

are $\mathbb{R}$-bounded, then $\Psi \in M_{p}^{p}(E)$.

A Banach space $E$ has a property $(\alpha)$ (see, e.g., [18]) if there exists a constant $\alpha$ such that

$$
\left\|\sum_{i, j=1}^{N} \alpha_{i j} \varepsilon_{i} \varepsilon_{j}^{\prime} x_{i j}\right\|_{L_{2}\left(\Omega \times \Omega^{\prime} ; E\right)} d y \leq \alpha\left\|\sum_{i, j=1}^{N} \varepsilon_{i} \varepsilon_{j}^{\prime} x_{i j}\right\|_{L_{2}\left(\Omega \times \Omega^{\prime} ; E\right)}
$$

for all $N \in \mathbb{N}, x_{i, j} \in E, \alpha_{i j} \in\{0,1\}, i, j=1,2, \ldots, N$, and all choices of independent, symmetric, $\{-1,1\}$-valued random variables $\varepsilon_{1}, \varepsilon_{2}, \ldots, \varepsilon_{N}, \varepsilon_{1}^{\prime}, \varepsilon_{2}^{\prime}, \ldots, \varepsilon_{N}^{\prime}$ on probability spaces $\Omega$, $\Omega^{\prime}$. For example the spaces $L_{p}(\Omega), 1 \leq p<\infty$, has the property $(\alpha)$.

Remark 1.2. If $E$ is UMD space with property $(\alpha)$ then these spaces satisfy the multiplier condition with respect to $p \in(1, \infty)$ (see [18]).

It is well known (see, e.g., [26]) that any Hilbert space satisfies the multiplier condition. There are, however, Banach spaces which are not Hilbert spaces but satisfy the multiplier condition, for example, UMD spaces (see $[11,12,18,36]$ ).

Definition 1.3. A positive operator $A$ is said to be $\mathbb{R}$-positive in the Banach space $E$ if there exists $\varphi \in(0, \pi]$ such that the set

$$
L_{A}=\left\{(1+|\xi|)(A+\xi I)^{-1}: \xi \in K_{\varphi}\right\}
$$

is $\mathbb{R}$-bounded.

Note that in Hilbert spaces every norm bounded set is $\mathbb{R}$-bounded. Therefore, in Hilbert spaces all positive operators are $\mathbb{R}$-positive. If $A$ is a generator of a contraction semigroup on $L_{q}, 1 \leq q \leq \infty$ [23], $A$ has bounded imaginary powers with $\left\|\left(-A^{i t}\right)\right\|_{B(E)} \leq$ $C e^{v|t|}, v<\pi / 2$ [14], or if $A$ is a generator of a semigroup with Gaussian bound [19] in $E \in$ $\mathrm{UMD}$, then this operator is $\mathbb{R}$-positive.

Let $\Omega \in \mathbb{R}^{n}$ and $l=\left(l_{1}, l_{2}, \ldots, l_{n}\right)$. Let $E_{0}$ and $E$ be two Banach spaces and $E_{0}$ continuously and densely embedded into $E$. Let us consider a Banach-valued function space $W_{p}^{l}\left(\Omega ; E_{0}, E\right)$ that consists of functions $u \in L_{p}\left(\Omega ; E_{0}\right)$ such that has the generalized derivatives $D_{k}^{l_{k}} u=\left(\partial^{l_{k}} / \partial x_{k}^{l_{k}}\right), u \in L_{p}(\Omega ; E)$, with norm

$$
\|u\|_{W_{p}^{l}\left(\Omega ; E_{0}, E\right)}=\|u\|_{L_{p}\left(\Omega ; E_{0}\right)}+\sum_{k=1}^{n}\left\|D_{k}^{l_{k}} u\right\|_{L_{p}(\Omega ; E)}<\infty .
$$

For $E_{0}=E$ the space $W_{p}^{l}\left(\Omega ; E_{0}, E\right)$ will be denoted by $W_{p}^{l}(\Omega ; E)$. For $\Omega=(a, b) \in \mathbb{R}$ and $l_{1}=l_{2}=\cdots=l_{n}=m$ the space $W_{p}^{l}\left(\Omega ; E_{0}, E\right)$ will be denoted by $W_{p}^{m}\left(a, b ; E_{0}, E\right)$.

By $\sigma_{\infty}(E)$ will be denoted a space of all compact operators in $E$. 


\section{Background materials}

Embedding theorems of vector-valued Sobolev spaces played important role in the present investigation. Embedding theorems in Banach-valued function spaces have been studied, for example, in $[6,25,29,31,33]$. This section concentrates on anisotropic Banach-valued Sobolev spaces $W_{p}^{l}\left(\Omega ; E_{0}, E\right)$ associated with Banach spaces $E_{0}, E$. Several conditions are found that ensure the continuity and compactness of embedding operators that are optimal regular in these spaces in terms of interpolations of $E_{0}$ and $E$. In particular, the most regular class of interpolation spaces $E_{\alpha}$ between $E_{0}, E$, depending on $\alpha$ and order of spaces are found that mixed derivatives $D^{\alpha}$ are bounded and compact from this space to $E_{\alpha}$-valued $L_{p}$ spaces. This results are generalized and improve the result of Lions and Peetre [25] for Banach-valued spaces and the embedding theorems for scalar Sobolev spaces [8, Section 9]. Multiplier theorems in the operator-valued $L_{p}$ spaces, are important tools in the theory of embedding of function spaces and PDE. Since the problems under consideration established the uniformly parameterized estimates, so we have to generalize multiplier theorems [18] for the case of $L_{p}$ multipliers depending on parameters. So, firstly by using a similar technique as [18] we show the following multiplier theorem.

Theorem 2.1. Let E be a UMD space with property $(\alpha)$ and let $\Psi_{h} \in C^{n}\left(\mathbb{R}^{n} /\{0\} ; B(E)\right)$ and there is some $C>0$ such that

$$
\sup _{x \in \mathbb{R}^{n} /\{0\},|\alpha| \leq n}\left\|D^{\alpha} \Psi_{h}(x)\right\|_{B(E)} \leq C
$$

for all $h=\left(h_{1}, h_{2}, \ldots, h_{L}\right) \in B(h)$. If

$$
\mathbb{R}\left\{\xi^{\beta} D_{\xi}^{\beta} \Psi_{h}(\xi): \xi \in V_{n}, \beta \in U_{n}\right\}=K_{\beta}<\infty
$$

uniformly with respect to $h$, then $\Psi_{h}(\xi)$ is a uniform collection of multipliers in $L_{p}\left(\mathbb{R}^{n} ; E\right)$.

If $n=1$, then the result remains true for $E$ without having the property $(\alpha)$.

Note 2.2. It is clear that Theorem 2.1 is valid for the case of multipliers without parameter and without assumption of the uniformly boundedness condition.

By virtue of [33] we obtain the following.

THeORem 2.3. Suppose the following conditions are satisfied:

(1) $E$ is a Banach space that satisfies the multiplier condition with respect to $p$ and $A$ is an $\mathbb{R}$-positive operator in $E$ for $\varphi$ with $0<\varphi \leq \pi$;

(2) $\alpha=\left(\alpha_{1}, \alpha_{2}, \ldots, \alpha_{n}\right), l=\left(l_{1}, l_{2}, \ldots, l_{n}\right)$ are $n$-tuples of nonnegative integer numbers such that

$$
\varkappa=|\alpha: l|=\sum_{k=1}^{n} \frac{\alpha_{k}}{l_{k}} \leq 1, \quad 1<p<\infty, 0<\mu \leq 1-\varkappa ;
$$

(3) $\Omega \in \mathbb{R}^{n}$ is a region such that there exists a bounded linear extension operator acting from $L_{p}(\Omega ; E)$ to $L_{p}\left(\mathbb{R}^{n} ; E\right)$ and also from $W_{p}^{l}(\Omega ; E(A), E)$ to $W_{p}^{l}\left(\mathbb{R}^{n} ; E(A), E\right)$. 
Then an embedding

$$
D^{\alpha} W_{p}^{l}(\Omega ; E(A), E) \subset L_{p}\left(\Omega ; E\left(A^{1-\varkappa-\mu}\right)\right)
$$

is continuous and there exists a positive constant $C_{\mu}$ such that

$$
\left\|D^{\alpha} u\right\|_{L_{p}\left(\Omega ; E\left(A^{1-\varkappa-\mu}\right)\right)} \leq C_{\mu}\left[h^{\mu}\|u\|_{W_{p}^{l}(\Omega ; E(A), E)}+h^{-(1-\mu)}\|u\|_{L_{p}(\Omega ; E)}\right]
$$

for all $u \in W_{p}^{l}(\Omega ; E(A), E)$ and $h$ with $0<h<h_{0}<\infty$.

THeorem 2.4. Suppose all conditions of Theorem 2.3 are satisfied and suppose $\Omega$ is a bounded region in $\mathbb{R}^{n}, A^{-1} \in \sigma_{\infty}(E)$. Then for $0<\mu<1-\varkappa$, an embedding

$$
D^{\alpha} W_{p}^{l}(\Omega ; E(A), E) \subset L_{p}\left(\Omega ; E\left(A^{1-\varkappa-\mu}\right)\right)
$$

is compact.

Indeed, putting $h=\|u\|_{L_{p}(\Omega ; E)} /\|u\|_{W_{p}^{l}(\Omega ; E(A), E)}$ in (1.12), we obtain a multiplicative inequality

$$
\left\|D^{\alpha} u\right\|_{L_{p}\left(\Omega ; E\left(A^{1-\varkappa-\mu}\right)\right)} \leq C_{\mu}\|u\|_{L_{p}(\Omega ; E)}^{\mu}\|u\|_{W_{p}^{l}(\Omega ; E(A), E)}^{1-\mu} .
$$

By virtue of [29] the embedding

$$
W_{p}^{l}(\Omega ; E(A), E) \subset L_{p}(\Omega ; E)
$$

is compact. Then from the estimate (2.7) we obtain assertion of Theorem 2.4.

By a similar manner as Theorem 2.3 we have the following.

Theorem 2.5. Suppose all conditions of Theorem 2.3 are satisfied.

Then for $0<\mu<1-\varkappa$ an embedding

$$
D^{\alpha} W_{p}^{l}(\Omega ; E(A), E) \subset L_{p}\left(\Omega ;(E(A), E)_{\varkappa, p}\right)
$$

is continuous and there exists a positive constant $C_{\mu}$ such that

$$
\begin{aligned}
& \left\|D^{\alpha} u\right\|_{L_{p}\left(\Omega ;(E(A), E)_{\varkappa+\mu, p}\right)} \\
& \quad \leq C_{\mu}\left[h^{\mu}\left(\|A u\|_{L_{p}(\Omega ; E)}+\sum_{k=1}^{n}\left\|D_{k}^{l_{k}} u\right\|_{L_{p}(\Omega ; E)}\right)+h^{-(1-\mu)}\|u\|_{L_{p}(\Omega ; E)}\right]
\end{aligned}
$$

for all $u \in W_{p}^{l}(\Omega ; E(A), E)$ and $h$ with $0<h<h_{0}<\infty$.

By a similar manner as Theorem 2.4 we have the following.

Theorem 2.6. Suppose all conditions of Theorem 2.3 are satisfied and suppose $\Omega$ is a bounded region in $\mathbb{R}^{n}, A^{-1} \in \sigma_{\infty}(E)$. Then for $0<\mu<1-\varkappa$ an embedding

$$
D^{\alpha} W_{p}^{l}(\Omega ; E(A), E) \subset L_{p}\left(\Omega ;(E(A), E)_{\varkappa+\mu, p}\right)
$$

is compact. 
Theorem 2.7 [32]. Let $E$ be a Banach space and $A$ a positive operator in E. Let $m$ be a positive integer, $1 \leq p<\infty$, and $1 / 2 p<\alpha<m+1 / 2 p$. Let $0 \leq \gamma<1$. Then for $\lambda \in S(\varphi)$ the operator $-A_{\lambda}^{1 / 2}$ generates a semigroup $e^{-A_{\lambda}^{1 / 2} x}$ which is holomorphic for $x>0$ and strongly continuous for $x \geq 0$. Moreover, there exists a constant $C>0$ such that for every $u \in(E$, $\left.E\left(A^{m}\right)\right)_{\alpha / m-(1+\gamma) / 2 p m, p}$ and $\lambda \in S(\varphi)$,

$$
\int_{0}^{\infty}\left\|A_{\lambda}^{\alpha} e^{-x A_{\lambda}^{1 / 2}} u\right\|_{E}^{p} x^{\gamma} d x \leq C\left(\|u\|_{\left(E, E\left(A^{m}\right)\right)_{\alpha / m-(1+\gamma) / 2 m p, p}^{p}}^{p}+|\lambda|^{p \alpha-(1+\gamma) / 2}\|u\|_{E}^{p}\right) .
$$

By using a similar techniques as [25] (or [35, Section 1.8.1]) we obtain the following.

TheOrem 2.8. Let the following conditions be satisfied:

(1) $l$ and $s$ are integer numbers, and $0 \leq s \leq l-1$;

(2) $\theta=(p s+1) / p l, x_{0} \in[0, b], 0<h \leq h_{0}, 0<\mu \leq 1-\theta, 1<p<\infty$;

Then, for $u \in W_{p}^{l}\left(0, b ; E_{0}, E\right)$, the following inequality holds:

$$
\left\|u^{(s)}\left(x_{0}\right)\right\|_{\left(E_{0}, E\right)_{\theta+\mu, p}} \leq h^{\mu}\|u\|_{W_{p}^{l}\left(0, b ; E_{0}, E\right)}+h^{-(1-\mu)}\|u\|_{L_{p}(0, b ; E)} .
$$

\section{Statement of problems}

Consider a nonlocal BVP for elliptic DOE

$$
\begin{gathered}
\sum_{k=1}^{n} a_{k}(x) D_{k}^{2} u(x)+A_{\lambda}(x) u(x)+\sum_{k=1}^{n} A_{k}(x) \frac{\partial u(x)}{\partial x_{k}}=f(x), \quad x \in G \subset \mathbb{R}^{n}, \\
L_{k j} u=\left[\alpha_{k j} u^{\left(m_{k j}\right)}\left(G_{k 0}\right)+\beta_{k j} u^{\left(m_{k j}\right)}\left(G_{k b}\right)\right]=0 \\
j=1,2, \quad k=1,2, \ldots, n,
\end{gathered}
$$

and nonlocal IBVP parabolic problem

$$
\begin{gathered}
\frac{\partial u(t, x)}{\partial t}+\sum_{k=1}^{n} a_{k}(x) D_{k}^{2} u(t, x)+A_{\lambda}(x) u(t, x)+\sum_{k=1}^{n} A_{k}(x) \frac{\partial u(t, x)}{\partial x_{k}}=f(t, x), \\
L_{k j} u=\left[\alpha_{k j} u^{\left(m_{k j}\right)}\left(t, G_{k 0}\right)+\beta_{k j} u^{\left(m_{k j}\right)}\left(t, G_{k b}\right)\right]=0, \quad u(0, x)=0, \\
j=1,2, \quad k=1,2, \ldots, n, t \in \mathbb{R}_{+}, x \in G \subset \mathbb{R}^{n},
\end{gathered}
$$

where

$$
\begin{gathered}
G=\left\{x=\left(x_{1}, x_{2}, \ldots, x_{n}\right), 0<x_{k}<b_{k},\right\}, \quad G_{+}=\mathbb{R}_{+} \times G, A_{\lambda}(x)=A(x)+\lambda, \\
G_{k 0}=\left(x_{1}, x_{2}, \ldots, x_{k-1}, 0, x_{k+1}, \ldots, x_{n}\right), \quad G_{k b}=\left(x_{1}, x_{2}, \ldots, x_{k-1}, b_{k}, x_{k+1}, \ldots, x_{n}\right), \\
m_{k} \in\{0,1\}, \quad D_{k}^{2}=\frac{\partial^{2}}{\partial x_{k}^{2}}, k=1,2, \ldots, n ;
\end{gathered}
$$

$\alpha_{j k}, \beta_{j k}, \delta_{j k i}$ are complex numbers, $a_{k}$ is real-valued function on $G$, and $A(x), A_{k}(x)$ for $x, y \in G$ are generally speaking, unbounded operators in $E$. 
We say that the elliptic problem (3.1) is a maximal $L_{p}$-regular, if for all $f \in L_{p}(G ; E)$ there exists a unique solution $u \in W_{p}^{2}(G ; E(A), E)$ of the problem (3.1) satisfying this problem almost everywhere and there exists a positive constant $C$ independent of $f$, such that has an estimate

$$
\sum_{k=1}^{n}\left\|D_{k}^{2} u\right\|_{L_{p}(G ; E)}+\|A u\|_{L_{p}(G ; E)} \leq C\|f\|_{L_{p}(G ; E)} .
$$

We say that the parabolic problem (3.2) is a maximal $L_{p}$-regular, if for all $f \in L_{p}\left(G_{+} ; E\right)$ there exists a unique solution $u$ satisfying the (3.2) problem almost everywhere and there exists a positive constant $C$ independent of $f$, such that has an estimate

$$
\left\|\frac{\partial u(t, x)}{\partial t}\right\|_{L_{p}\left(G_{+} ; E\right)}+\|A u\|_{L_{p}\left(G_{+} ; E\right)} \leq C\|f\|_{L_{p}\left(G_{+} ; E\right)} .
$$

\section{Ordinary DOE with constant coefficients}

Let us first consider a nonlocal and nonhomogenous boundary value problem for ordinary DOE

$$
\begin{gathered}
(L+\lambda) u=a u^{\prime \prime}(x)+A_{\lambda} u(x)=f(x), \quad x \in(0, b), \\
L_{k} u=\alpha_{k} u^{\left(m_{k}\right)}(0)+\beta_{k} u^{\left(m_{k}\right)}(b)+\sum_{j=1}^{N_{k}} \delta_{k j} u^{\left(m_{k}\right)}\left(x_{k j}\right)=f_{k}, \quad k=1,2,
\end{gathered}
$$

where $f_{k} \in E_{k}=(E(A), E)_{\theta_{k}, p}, \theta_{k}=m_{k} / 2+1 / 2 p, p \in(1, \infty), m_{k} \in\{0,1\} ; a, \alpha_{k}, \beta_{k}, \delta_{k j}$, are complex numbers and $x_{k j} \in(0, b)$; $A$ is a possible unbounded operator in $E$. Let $\omega_{j}$, $j=1,2$, be roots of the equation

$$
a \omega^{2}+1=0
$$

Condition 4.1. Let the following conditions be satisfied:

(1) $A$ is a positive operator in a Banach space $E$ for $\varphi \in(0, \pi / 2)$;

(2) $a \neq b^{2}$ for all $b \in \mathbb{R}$;

(3) $\eta=(-1)^{m_{1}} \alpha_{1} \beta_{2}-(-1)^{m_{2}} \alpha_{2} \beta_{1} \neq 0$.

Consider the problem

$$
\begin{gathered}
(L+\lambda) u=a u^{\prime \prime}(x)+(A+\lambda) u(x)=0 \\
L_{k} u=\left[\alpha_{k} u^{\left(m_{k}\right)}(0)+\beta_{k} u^{\left(m_{k}\right)}(b)+\sum_{j=1}^{N_{k}} \delta_{k j} u^{\left(m_{k}\right)}\left(x_{k j}\right)\right]=f_{k}, \quad k=1,2 .
\end{gathered}
$$

Lemma 4.2. Let Condition 4.1 be satisfied. Then the problem (4.3)-(4.4) for $f_{k} \in E_{k}, \lambda \in$ $S(\varphi)$, and sufficiently large $|\lambda|$ has a unique solution $u$ that belongs to $W_{p}^{2}(0, b ; E(A), E)$ and 
the coercive uniform estimate

$$
\sum_{i=0}^{2}|\lambda|^{1-i / 2}\left\|u^{(i)}\right\|_{L_{p}}+\|A u\|_{L_{p}} \leq M \sum_{k=1}^{2}\left(\left\|f_{k}\right\|_{E_{k}}+|\lambda|^{1-\theta_{k}}\left\|f_{k}\right\|\right)
$$

holds with respect to parameter $\lambda$.

Proof. From conditions (1.12) and (1.13), by virtue of [39, Lemma 5.3.2/1], for $\lambda \in S\left(\varphi_{0}\right)$, there exists the holomorphic for $x>0$ and strongly continuous for $x \geq 0$ semigroups $e^{x \omega_{1} A_{\lambda}^{1 / 2}}, e^{-(b-x) \omega_{2} A_{\lambda}^{1 / 2}}$, and the arbitrary solution of $(4.3)$, belonging to space $W_{p}^{2}(0, b ; E(A)$, $E)$, has a form

$$
u(x)=e^{x \omega_{1} A_{\lambda}^{1 / 2}} g_{1}+e^{-(b-x) \omega_{2} A_{\lambda}^{1 / 2}} g_{2}
$$

where

$$
A_{\lambda}=A+\lambda I, g_{k} \in(E(A), E)_{1 / 2 p, p}, \quad k=1,2
$$

By taking into account boundary conditions (4.4) we obtain algebraic linear equations with respect to $g_{1}, g_{2}$;

$$
\begin{aligned}
(-1)^{m_{k}} & {\left[\alpha_{k}+\beta_{k} e^{-b \omega_{1} A_{\lambda}^{1 / 2}}+\sum_{j=1}^{N_{k}} \delta_{k j} e^{-x_{k j} \omega_{1} A_{\lambda}^{1 / 2}}\right] A_{\lambda}^{m_{k} / 2} g_{1} } \\
& +\left[\alpha_{k} e^{-b \omega_{2} A_{\lambda}^{1 / 2}}+\beta_{k}+\sum_{j=1}^{N_{k}} \delta_{k j} e^{-\left(b-x_{k j}\right) \omega_{2} A_{\lambda}^{1 / 2}}\right] A_{\lambda}^{m_{k} / 2} g_{2}=f_{k}, \quad k=1,2 .
\end{aligned}
$$

A system (4.8) is matrix-operator equations. Let $D(\lambda)$ be a main operator determinant of (4.8). By virtue of the properties of positive operators and holomorphic semigroups [35, Section 1.14] it is clear to see that $\|D(\lambda)\|_{B\left(E^{2}\right)} \rightarrow 0$ for $|\lambda| \rightarrow \infty$. Then by conditions $\eta \neq 0$ and $\lambda \in S(\varphi), \lambda \rightarrow \infty$, the operator-matrix $Q(\lambda)=[\theta+D(\lambda)]^{-1}$ is invertible and bounded uniformly with respect to the parameters $\lambda$. Consequently, the system (4.8) has a unique solution for $\lambda \in S(\varphi)$ and sufficiently large $|\lambda|$. From the expressions of operators $D(\lambda)$ and $Q(\lambda)$ it follows that these operators are bounded, and operators containing the expression $D(\lambda)$ are commuting with any powers of operators $A_{\lambda}^{1 / 2}$. Consequently, substituting the values of $g_{1}, g_{2}$ into (4.8), we obtain a representation of the solution of the problem (4.3)-(4.4):

$$
u(x)=\sum B_{1 j}(\lambda) U_{1 \lambda}\left(x_{j}\right) f_{1}+B_{2 j}(\lambda) U_{2 \lambda}\left(x_{j}\right) f_{2},
$$

where $B_{k j}(\lambda)$ are bounded operators in $E$ uniformly with respect to $\lambda$ and

$$
U_{1 \lambda}(x)=e^{x \omega_{1} A_{\lambda}^{1 / 2}}, \quad U_{2 \lambda}(x)=e^{-(b-x) \omega_{1} A_{\lambda}^{1 / 2}}, \quad x_{j} \in[0, b] .
$$

By virtue of Theorem 2.7, the properties of holomorphic semigroups, in view of uniformly boundedness of operator $Q(\lambda)$, and the representation of the solution (4.9), we obtain the estimate (4.5). 
Theorem 4.3. Let Condition 4.1 be satisfied for $\varphi \in(0, \pi / 3)$. Let $E$ be a Banach space satisfying the multiplier condition with respect to $p \in(1, \infty)$ and $A$ an $\mathbb{R}$-positive operator in $E$. Then an operator $u \rightarrow D(\lambda) u=\left\{L(\lambda) u, L_{1} u, L_{2} u\right\}$ for $\lambda \in S(\varphi)$ and sufficiently large $|\lambda|$ is an isomorphism from $W_{p}^{2}(0, b ; E(A), E)$ onto $L_{p}(0, b ; E)+E_{1}+E_{2}$. Moreover, the coercive uniform estimate

$$
\sum_{j=0}^{2}|\lambda|^{1-j / 2}\left\|u^{(j)}\right\|_{L_{p}}+\|A u\|_{L_{p}} \leq C\left[\|f\|_{L_{p}}+\sum_{k=1}^{2}\left(\left\|f_{k}\right\|_{E_{k}}+|\lambda|^{1-\theta_{k}}\left\|f_{k}\right\|_{E}\right)\right]
$$

holds with respect to parameter $\lambda$.

Proof. We have proved the uniqueness of the solution of the problem (4.1) in Lemma 4.2. Let

$$
\bar{f}(x)= \begin{cases}f(x) & \text { if } x \in[0, b] \\ 0 & \text { if } x \notin[0, b]\end{cases}
$$

We now show that a solution of the problem (4.1) which belongs to space $W_{p}^{2}(0, b$; $E(A) E)$ can be represented as a sum $v(x)=u_{1}(x)+u_{2}(x)$, where $u_{1}$ is a restriction on $[0, \mathrm{~b}]$ of a solution $u$ of an equation

$$
L(\lambda) u=\bar{f}(x), \quad x \in \mathbb{R}=(-\infty, \infty),
$$

and $u_{2}$ is a solution of a problem

$$
L(\lambda) u=0, \quad L_{k} u=f_{k}-L_{k} u_{1}
$$

The solution of (4.13) is given by formula

$$
u(x)=F^{-1} L^{-1}(\lambda, \xi) F \bar{f}=\frac{1}{2 \pi} \int_{-\infty}^{\infty} e^{i \xi x} L^{-1}(\lambda, \xi)(F \bar{f})(\xi) d \xi
$$

where $F \bar{f}$ is a Fourier transform of a function $\bar{f}$, and

$$
L(\lambda, \xi)=\left(-a \xi^{2}+\lambda\right) I+A
$$

Due to $\mathbb{R}$-positivity of operator $A$ and by virtue of Kahane's contraction principle, we obtain

$$
\begin{gathered}
\mathbb{R}\left(\left\{\xi^{\beta} D_{\xi}^{\beta} A L^{-1}(\lambda, \xi): \beta \in\{0,1\}, \xi \neq 0\right\}\right) \leq M, \\
\mathbb{R}\left(\left\{\xi^{\beta} D_{\xi}^{\beta}|\lambda|^{1-j / 2} \xi^{j} L^{-1}(\lambda, \xi): \beta \in\{0,1\}, \xi \neq 0, j=0,1,2\right\}\right) \leq M_{\beta} .
\end{gathered}
$$


Then in view of Definition 1.1 it follows from (4.17) that the operator-valued functions $A L^{-1}(\lambda, \xi),|\lambda|^{1-j / 2} \xi^{j} L^{-1}(\lambda, \xi), j=0,1,2$, are uniformly bounded Fourier multipliers in $L_{p}(\mathbb{R} ; E)$. Therefore, we obtain that the problem $(4.13)$ has a solution $u_{0} \in W_{p}^{2}(\mathbb{R}$; $E(A), E)$ and

$$
\sum_{j=0}^{2}|\lambda|^{1-j / 2}\left\|u_{0}^{(j)}\right\|_{L_{p}(\mathbb{R} ; E)}+\left\|A u_{0}\right\|_{L_{p}(\mathbb{R} ; E)} \leq C\|\bar{f}\|_{L_{p}(\mathbb{R} ; E)}
$$

So, we obtain that $u_{1} \in W_{p}^{2}(0, b ; E(A), E)$ is the solution of $(4.13)$ on $(0, b)$. By virtue of [25] we get that

$$
u^{\left(m_{k}\right)}(\cdot) \in(E(A) ; E)_{\theta_{k}, p}, \quad k=1,2
$$

Hence, $L_{k} u_{1} \in E_{k}$. Thus by virtue of Theorem 4.3 the problem (4.14) has a unique solution $u_{2}(x)$ that belongs to space $W_{p}^{2}(0, b ; E(A), E)$, and for sufficiently large $|\lambda|$ we have

$$
\begin{aligned}
\sum_{j=0}^{2}|\lambda|^{1-j / 2}\left\|u_{2}^{(j)}\right\|_{L_{p}(\mathbb{R} ; E)}+\left\|A u_{2}\right\|_{L_{p}(\mathbb{R} ; E)} \\
\leq C \sum_{k=1}^{2}\left[\left\|f_{k}\right\|_{E_{k}}+|\lambda|^{1-\theta_{k}}\left\|f_{k}\right\|_{E}+|\lambda|^{1-\theta_{k}}\left\|L_{k} u_{1}\right\|_{E}\right. \\
\left.+\left\|u_{1}^{\left(m_{k}\right)}\right\|_{C\left([0, b] ; E_{k}\right)}+\left|\lambda^{1-\theta_{k}}\right|\|u\|_{C([0, b] ; E)}\right] .
\end{aligned}
$$

From (4.18) for $\lambda \in S(\varphi)$ we obtain

$$
\sum_{j=0}^{2}|\lambda|^{1-j / 2}\left\|u_{1}^{(j)}\right\|_{L_{p}}+\left\|A u_{1}\right\|_{L_{p}} \leq C\|f\|_{L_{p}}
$$

Therefore, by virtue of [25] and by the estimate (4.21) for $x_{0} \in[0, b]$, we have

$$
\left\|u_{1}^{\left(m_{k}\right)}\left(x_{0}\right)\right\|_{E_{k}} \leq C\left\|u_{1}\right\|_{W_{p t}^{2}(0, b ; E(A), E)} \leq C\|f\|_{L_{p}(0, b ; E)} .
$$

By virtue of Theorem 2.8 for $\lambda=\mu^{2}, u \in W_{p}^{2}(0, b ; E)$, we have

$$
|\mu|^{2-m_{k}}\left\|u^{\left(m_{k}\right)}\left(x_{0}\right)\right\|_{E} \leq C\left[|\mu|^{1 / p}\|u\|_{W_{p}^{2}(0, b ; E)}+|\mu|^{2+1 / p}\|u\|_{L_{p}}\right] .
$$

Hence from estimates (4.20), (4.22), and (4.23) we obtain

$$
\sum_{j=0}^{2}|\lambda|^{1-j / 2}\left\|u_{2}^{(j)}\right\|_{L_{p}}+\left\|A u_{2}\right\|_{L_{p}} \leq C\left(\|f\|_{L_{p}}+\sum_{k=1}^{2}\left(\left\|f_{k}\right\|_{E_{k}}+|\lambda|^{1-\theta_{k}}\left\|f_{k}\right\|_{E}\right)\right) .
$$

Then estimates (4.21) and (4.24) imply (4.11). 
Consider a BVP

$$
\begin{gathered}
L u=a u^{\prime \prime}(x)+A u(x)=f(x), \quad x \in(0, b), \\
L_{k} u=\alpha_{k} u^{\left(m_{k}\right)}(0)+\beta_{k} u^{\left(m_{k}\right)}(b)+\sum_{j=1}^{N_{k}} \delta_{k j} u^{\left(m_{k}\right)}\left(x_{k j}\right)=0, \quad k=1,2 .
\end{gathered}
$$

Let $B$ denote a differential operator in $L_{p}(0, b ; E)$ generating by BVP $(4.25)-(4.26)$, that is,

$$
\begin{gathered}
D(B)=W_{p}^{2}\left(0, b ; E(A), E, L_{k}\right), \\
B u=a u^{(2)}(x)+A u(x), \quad x \in(0, b) .
\end{gathered}
$$

Theorem 4.4. Let all conditions of Theorem 4.3 hold for $\varphi \in(0, \pi / 3)$. Then the operator $B$ is positive in $L_{p}(0, b ; E)$.

Proof. From (1.12)-(1.13), part of Condition 4.1, we have

$$
(L+\lambda)=a\left(\frac{d}{d t}-\omega_{1} A_{\lambda}\right)\left(\frac{d}{d t}-\omega_{2} A_{\lambda}\right)
$$

By using the above representation and by using a similar technique as [39, Lemma 5.3.2/1] we obtain that $\omega_{k} A_{\lambda}, k=1,2$, for $\lambda \in S(\varphi)$ are generators of the bounded analytic $C_{0}$-semigroups $U_{1 \lambda}(x)=e^{x \omega_{1} A_{\lambda}^{1 / 2}}, U_{2 \lambda}(x)=e^{-(b-x) \omega_{2} A_{\lambda}^{1 / 2}}$ in $E$ and a solution of $(4.11)$ is represented as

$$
u(x)=U_{1 \lambda}(x) g_{1}+U_{2 \lambda}(x) g_{2}+\int_{0}^{b} U_{0 \lambda}(x-y) f(y) d y, \quad g_{k} \in E,
$$

where

$$
U_{0 \lambda}(x-y)= \begin{cases}-a^{-1} A_{\lambda}^{-1}\left(\omega_{2}-\omega_{1}\right)^{-1} U_{1 \lambda}(x-y), & x \geq y, \\ a^{-1} A_{\lambda}^{-1}\left(\omega_{2}-\omega_{1}\right)^{-1} U_{2 \lambda}(x-y), & x \leq y .\end{cases}
$$

By taking into account boundary conditions (4.26) we obtain from (4.29) a representation of the solution of problem (4.25)-(4.26):

$$
\begin{gathered}
u(x)=\int_{0}^{b} G_{\lambda}(x, y) f(y) d y, \\
G_{\lambda}(x, y)=\sum B_{1 j}(\lambda) U_{1 \lambda}(y)+B_{2 j}(\lambda) U_{2 \lambda}(y)+U_{0 \lambda}(x-y),
\end{gathered}
$$

where $B_{k j}(\lambda)$ are bounded operators in $E$ uniformly with respect to $\lambda$. Due to holomorphic semigroups of $U_{k \lambda}$, we have (see, e.g., [35, Section 1.14])

$$
\begin{gathered}
\left\|U_{1 \lambda}(x)\right\| \leq C_{1} e^{-\nu_{1} x}, \quad\left\|U_{2 \lambda}(x)\right\| \leq C_{2} e^{-\nu_{2}(b-x)}, \\
\left\|U_{0 \lambda}(x-y)\right\| \leq C_{0} \frac{e^{-\nu_{0}|x-y|}}{|x-y|}, \quad C_{i}, \nu_{i} \in \mathbb{R}_{+}, \quad i=0,1,2 .
\end{gathered}
$$


So in view of the representation (4.31), by virtue of the above estimate, by using [12, Lemma 7.1] and a similar technique as [12, Theorem 7.4], we obtain the positivity of operator $B$.

Remark 4.5. If $a$ is a real negative number then (1.13), part of Condition 4.1, is satisfied for $0<\varphi \leq \pi$ and Theorems 4.3, 4.4 are valid for $0<\varphi \leq \pi$.

\section{Partial DOE with constant coefficients}

$$
\begin{gathered}
\sum_{k=1}^{n} a_{k} D_{k}^{2} u(x)+A_{\lambda} u(x)=f(x), \quad x \in G, \\
L_{k j} u=\left[\alpha_{k j} u^{\left(m_{k j}\right)}\left(G_{k 0}\right)+\beta_{k j} u^{\left(m_{k j}\right)}\left(G_{k b}\right)\right] \\
+\sum_{i=1}^{N_{k j}} \delta_{k j i} u^{\left(m_{k j}\right)}\left(G_{k i}\right)=0, \quad j=1,2, k=1,2, \ldots, n,
\end{gathered}
$$

$\alpha_{k}, \beta_{k}, \delta_{k j}$ are complex numbers, $a_{k}$ are complex numbers, and $A$ is, generally speaking, an unbounded operator in $E$ and

$$
G_{k i}=\left(x_{1}, x_{2}, \ldots, x_{k-1}, x_{k i}, x_{k+1}, \ldots, x_{n}\right), \quad x_{k i} \in\left(0, b_{k}\right) .
$$

Condition 5.1. Let the following conditions be satisfied:

(1) $E$ is a Banach space satisfying multiplier condition with respect to $p \in(1, \infty)$;

(2) $a_{k}(x) \neq b_{k}^{2}$, where $b_{k} \in \mathbb{R}$;

(3) $\eta_{k}=(-1)^{m_{1}} \alpha_{k 1} \beta_{k 2}-(-1)^{m_{2}} \alpha_{k 2} \beta_{k 1} \neq 0, k=1,2, \ldots, n$.

Theorem 5.2. Let Condition 5.1 be satisfied and $A$ is an $\mathbb{R}$-positive operator in $E$. Then

(a) the problem (5.1)-(5.2) for $f \in L_{p}(G ; E), \lambda \in S(\varphi)$, and for sufficiently large $|\lambda|$ has a unique solution that belongs to the space $W_{p}^{2}(G ; E(A), E)$ and the coercive uniform estimate

$$
\sum_{k=1}^{n} \sum_{i=0}^{2}(1+|\lambda|)^{1-i / 2}\left\|D_{k}^{i} u\right\|_{L_{p}(G ; E)}+\|A u\|_{L_{p}(G ; E)} \leq M\|f\|_{L_{p}(G ; E)}
$$

holds with respect to parameter $\lambda$;

(b) the operator $L_{0}$, generated by $B V P(5.1)-(5.2)$, is positive in $L_{p}(G ; E)$.

Proof. Let us first consider a nonlocal boundary value problem for ordinary DOE

$$
\begin{gathered}
L u=a_{1} u^{(2)}\left(x_{1}\right)+A_{\lambda} u\left(x_{1}\right)=f\left(x_{1}\right), \quad x_{1} \in\left(0, b_{1}\right), \\
L_{1 j} u=\left[\alpha_{1 j} u^{\left(m_{1 j}\right)}(0)+\beta_{1 j} u^{\left(m_{1 j}\right)}\left(b_{1}\right)\right]+\sum_{i=1}^{N_{1 j}} \delta_{1 j i} u^{\left(m_{1 j}\right)}\left(x_{1 i}\right)=0, \\
x_{1 i} \in\left(0, b_{1}\right), \quad j=1,2,
\end{gathered}
$$

in $L_{p}\left(0, b_{1} ; E\right)$, where $A$ is a positive operator in $E$ and $\alpha_{1 j}, \beta_{1 j}, \delta_{1 j i}$ are complex numbers. 
By virtue of Theorem 4.3 we obtain that for all $f \in L_{p}\left(0, b_{1} ; E\right), \lambda \in S(\varphi)$, and sufficiently large $|\lambda|$ the problem (5.5) has a unique solution that belongs to the space $W_{p}^{2}\left(0, b_{1} ; E(A), E\right)$, and coercive uniformity is defined by

$$
\sum_{i=0}^{2}(1+|\lambda|)^{1-i / 2}\left\|u^{(i)}\right\|_{p}+\|A u\|_{p} \leq M\|f\|_{p}
$$

with respect to $\lambda$; the estimate holds for the solution of the problem (5.5). Consider in $L_{p}\left(G_{2} ; E\right)$ a BVP

$$
\begin{gathered}
\sum_{k=1}^{2} a_{k} D_{k}^{2} u(x)+(A+\lambda) u(x)=f(x), \\
L_{k j} u=\left[\alpha_{k j} u^{\left(m_{k j}\right)}\left(G_{k 0}\right)+\beta_{k j} u^{\left(m_{k j}\right)}\left(G_{k b}\right)\right]+\sum_{i=1}^{N_{k j}} \delta_{k j i} u^{\left(m_{k j}\right)}\left(G_{k i}\right)=0, \quad j, k=1,2,
\end{gathered}
$$

where $G_{2}=\left(0, b_{1}\right) \times\left(0, b_{2}\right)$. It is clear that

$$
L_{p}\left(G_{2} ; E\right)=L_{p}\left(0, b_{2} ; L_{p}\left(0, b_{1} ; E\right)\right) .
$$

The problem (5.7) can be expressed in the following view:

$$
D_{2}^{2} u\left(x_{2}\right)+B u\left(x_{2}\right)+\lambda u\left(x_{2}\right)=f\left(x_{2}\right), \quad L_{2 j} u=0,
$$

where $B$ is a differential operator in $L_{p}\left(0, b_{1} ; E\right)$ generated by problem (5.5). It is known (see, e.g., $[10,11])$ due to $E \in U M D, p \in(1, \infty)$, that the space $L_{p}\left(0, b_{1} ; E\right)$ is UMD space. Moreover, by virtue of Theorem 4.4 operator $B$ is $\mathbb{R}$-positive in $L_{p}\left(0, b_{1} ; E\right)$. Then again applying Theorem 4.3 we obtain that for all $f \in L_{p}\left(G_{2} ; E\right), \lambda \in S(\varphi)$, and sufficiently large $|\lambda|$ the problem (5.9), that is, the problem (5.7), has a unique solution that belongs to the space $W_{p}^{2}\left(G_{2} ; E(A), E\right)$, and the coercive uniform estimate

$$
\sum_{i=0}^{2}(1+|\lambda|)^{1-(i / 2)}\left\|D_{2}^{i}(B+\lambda)^{-1} u\right\|_{L_{p}\left(G_{2} ; E\right)}+\|B u\|_{L_{p}\left(G_{2} ; E\right)} \leq M\|f\|_{L_{p}\left(G_{2} ; E\right)}
$$

holds with respect to $\lambda$. Moreover, the estimate (5.6) implies

$$
\|B u\|_{L_{p}\left(0, b_{1} ; E\right)} \sim\|u\|_{W_{p}^{2}\left(0, b_{1}, E(A), E\right)} .
$$

Then estimates (5.10) and (5.11) imply the assertion of Theorem 4.3 for $n=2$. By expending this process we obtain the assertion (a). The assertion (b) is obtained by using Theorem 4.4.

5.1. Partial DOE with variable coefficients. Consider the boundary value problem (3.1).

Theorem 5.3. Let Condition 5.1 be satisfied for all $x \in G$ and

(1) $A(x)$ is an $\mathbb{R}$ positive in $E$ uniformly with respect to $x$ and $A\left(G_{0 k}\right)=A\left(G_{b k}\right), a_{k}(x)$ are continuous functions on $\bar{G}$ such that $a_{k}\left(G_{j 0}\right)=a_{k}\left(G_{j b}\right), k, j=1,2, \ldots, n$; 
(2) $A(x) A^{-1}\left(x^{0}\right) \in C(G ; B(E))$ and for any $\varepsilon>0$, for a.e. $x \in G$, and for $u \in(E(A), E)_{1 / 2, \infty}$,

$$
\left\|A_{k}(x) u\right\| \leq \varepsilon\|u\|_{(E(A), E)_{1 / 2, \infty}}+C(\varepsilon)\|u\| .
$$

Then

(a) the problem (3.1) for $f \in L_{p}(G ; E), \lambda \in S(\varphi)$, and for sufficiently large $|\lambda|$ has a unique solution that belongs to the space $W_{p}^{2}(G ; E(A), E)$. And the coercive uniform estimate

$$
\sum_{k=1}^{n} \sum_{i=0}^{2}(1+|\lambda|)^{1-i / 2}\left\|D_{k}^{i} u\right\|_{L_{p}(G ; E)}+\|A u\|_{L_{p}(G ; E)} \leq M\|f\|_{L_{p}(G ; E)}
$$

holds with respect to parameter $\lambda$;

(b) the operator $O$ generating by BVP (3.1) is positive in $L_{p}(G ; E)$.

Proof. Let $G_{1}, G_{2}, \ldots, G_{N}$ be regions in $\mathbb{R}$ and $\varphi_{1}, \varphi_{2}, \ldots, \varphi_{N}$ be corresponding a partition of unique that, functions $\varphi_{j}$ are smooth on $\mathbb{R}, \sigma_{j}=\operatorname{supp} \varphi_{j} \subset G_{j}$ and $\sum_{j=1}^{N} \varphi_{j}(x)=1$. Then for all $u \in W_{p}^{2}(G ; E(A), E)$ we have $u(x)=\sum_{j=1}^{N} u_{j}(x)$, where $u_{j}(x)=u(x) \varphi_{j}(x)$. Moreover, due to the nonlocalness of boundary conditions, functions $\varphi_{j}$ are chosen such that $\sigma_{j}=\operatorname{supp} \varphi_{j}$ adjoin with boundary $G_{k 0}, G_{k b}$ and consist of the sets; $\sigma_{j k 0}$ and $\sigma_{j k b}$ that is, $\tilde{\sigma}_{j k}=\sigma_{j k 0} \cup \sigma_{j k b}$, where $\sigma_{j k 0}$ are parts of $\tilde{\sigma}_{j k}$ adjoin with $G_{k 0}$ and $\sigma_{j k b}$ is the part of $\tilde{\sigma}_{j k}$ adjoin with $G_{k b}$. Let us consider the case when the regions $G_{j}$ adjoin with the boundary points and contain $\tilde{\sigma}_{j m}$. Let $u \in W_{p}^{2}(G ; E(A), E)$. Then from (3.1) we obtain

$$
(L+\lambda) u_{j}=\sum_{k=1}^{n} a_{k} D_{k}^{2} u_{j}(x)+A_{\lambda}(y) u_{j}(x)=f_{j}(x)
$$

where

$$
f_{j}=f \varphi_{j}+\sum_{k=1}^{n} a_{k}\left[2 \frac{\partial u}{\partial x_{k}} \frac{\partial \varphi_{j}}{\partial x_{k}}+u \frac{\partial^{2} \varphi_{j}}{\partial x_{k}^{2}}\right]-\sum_{k=1}^{n} \varphi_{j} A_{k}(x) \frac{\partial u(x)}{\partial x_{k}}, \quad j=1,2, \ldots, N
$$

Let $\operatorname{supp} \varphi_{j}$ partially belong to $G$. Freezing coefficients in (5.14) obtain that

$$
\sum_{k=1}^{n} a_{k}\left(x_{j}\right) D_{k}^{2} u_{j}(x)+A_{\lambda}\left(x_{j}\right) u_{j}(x)=F_{j}(x)
$$

where

$$
F_{j}=f_{j}+\left[A\left(x_{j}\right)-A(x)\right] u_{j}+\sum_{k=1}^{n}\left[a_{k}(x)-a\left(x_{j}\right)\right] D_{k}^{2} u_{j}(x)
$$

Suppose functions $\varphi_{j}(x)$ such that $L_{m k} u_{j}=0, m=1,2$, for all $u \in W_{p}^{2}(G ; E(A), E)$. Since functions $u_{j}(x)$ have the compact supports, then extending $u_{j}(x)$ on outsides of 
$\operatorname{supp} \varphi_{j}$ from (5.16) we obtain boundary value problems with constant coefficients:

$$
\begin{gathered}
\sum_{k=1}^{n} a_{k}\left(x_{j}\right) D_{k}^{2} u_{j}(x)+A_{\lambda}\left(x_{j}\right) u_{j}(x)=F_{j}(x), \\
L_{m k} u_{j}=0, \quad m=1,2, j=1,2, \ldots, N .
\end{gathered}
$$

By using Theorem 5.2 we obtain that the problem (5.18) has a unique solution $u_{j}$ and for $\lambda \in S(\varphi)$ and sufficiently large $|\lambda|$ the following coercive estimate:

$$
\sum_{k=1}^{n} \sum_{i=0}^{2}|\lambda|^{1-i / 2}\left\|D_{k}^{i} u_{j}\right\|_{G_{j}, p}+\left\|A u_{j}\right\|_{G_{j}, p} \leq C\left\|F_{j}\right\|_{G_{j}, p}
$$

holds. Whence, using properties of the smoothness of coefficients of (5.15), (5.17) and choosing diameters of $\sigma_{j}$ sufficiently small, we get that

$$
\left\|F_{j}\right\|_{G_{j}, p} \leq \varepsilon|| u_{j}\left\|_{W_{p}^{2}\left(G_{j} ; E(A), E\right)}+C(\varepsilon)\right\| u_{j} \|_{G_{j}, p},
$$

where $\varepsilon$ is a sufficiently small and $C(\varepsilon)$ is a continuous function. Consequently, from (5.19) and (5.20), we get

$$
\begin{gathered}
\sum_{k=1}^{n} \sum_{i=0}^{2}|\lambda|^{1-i / 2}\left\|D_{k}^{i} u_{j}\right\|_{G_{j}, p}+\left\|A u_{j}\right\|_{G_{j}, p} \\
\leq C\|f\|_{G_{j}, p}+\varepsilon\left\|u_{j}\right\|_{W_{p}^{2}}+C(\varepsilon)\left\|u_{j}\right\|_{G_{j}, p} .
\end{gathered}
$$

Choosing $\varepsilon<1$ from the above inequality, we have

$$
\sum_{k=1}^{n} \sum_{i=0}^{2}|\lambda|^{1-i / 2}\left\|D_{k}^{i} u_{j}\right\|_{G_{j}, p}+\left\|A u_{j}\right\|_{G_{j}, p} \leq C\left[\|f\|_{G_{j}, p}+\left\|u_{j}\right\|_{G_{j}, p}\right] .
$$

By a similar manner we also obtain estimates (5.22) for regions $G_{j}$ entirely belonging to $G$. Then using the equality $u(x)=\sum_{j=1}^{N} u_{j}(x)$ and by virtue of the estimate (5.22) for $u \in W_{p}^{2}(G ; E(A), E)$, we have

$$
\sum_{k=1}^{n} \sum_{i=0}^{2}|\lambda|^{1-i / 2}\left\|D_{k}^{i} u_{j}\right\|_{p}+\left\|A u_{j}\right\|_{p} \leq C\left[\|(L+\lambda) u\|_{p}+\|u\|_{p}\right] .
$$

Let $u \in W_{p}^{2}(G ; E(A), E)$ be a solution of the problem (3.1). Then for $\lambda \in S(\varphi)$, we have

$$
\|u\|_{p}=\|(L+\lambda) u-L u\|_{p} \leq \frac{1}{\lambda}\left[\|(L+\lambda) u\|_{p}+\|u\|_{W_{p}^{2}}\right] .
$$

Then by embedding Theorems 2.3, 2.5 and by virtue of (5.23), (5.24) for sufficiently large $|\lambda|$ we have

$$
\sum_{k=1}^{n} \sum_{i=0}^{2}|\lambda|^{1-i / 2}\left\|D_{k}^{i} u_{j}\right\|_{p}+\left\|A u_{j}\right\|_{p} \leq C\|(L+\lambda) u\|_{p}
$$


Let us consider an operator $O_{\lambda}$, acting in $L_{p}(G ; E)$, that is generated by the problem (3.1), that is,

$$
\begin{gathered}
D\left(O_{\lambda}\right)=W_{p}^{2}\left(G ; E(A), E, L_{k m}\right) \\
O_{\lambda} u=\sum_{k=1}^{n} a_{k}(x) D_{k}^{2} u(x)+A_{\lambda}(x) u(x)+\sum_{k=1}^{n} A_{k}(x) \frac{\partial u(x)}{\partial x_{k}} .
\end{gathered}
$$

The estimate (5.25) implies that the problem (3.1) has only a unique solution and the operator $O_{\lambda}$ has an invertible operator in its rank space. We need to show that this rank space coincide with the space $L_{p}(0, b ; E)$. We consider the smooth functions $g_{j}=g_{j}(x)$ with respect to the partition of the unique $\varphi_{j}=\varphi_{j}(y)$ on the region $G$ that equals one on $\operatorname{supp} \varphi_{j}$, where supp $g_{j} \subset G_{j}$ and $\left|g_{j}(x)\right|<1$. Let us construct for all $j$ the function $u_{j}$, that is defined on the regions $\Omega_{j}=G \cap G_{j}$ and satisfying the problem (3.1). Consider at first when $G_{j}$ adjoin to the boundary points. The problem (3.1) can be expressed in the form

$$
\begin{aligned}
\sum_{k=1}^{n} a_{k}\left(x_{j}\right) D_{k}^{2} u_{j}(x)+A_{\lambda}\left(x_{j}\right) u_{j}(x) & \\
= & g_{j}\left\{f+\left[A\left(x_{j}\right)-A(x)\right] u_{j}+\sum_{k=1}^{n}\left[a_{k}(x)-a_{k}\left(x_{j}\right)\right] D_{k}^{2} u_{j}-\sum_{k=1}^{n} A_{k}(x) \frac{\partial u_{j}(x)}{\partial x_{k}}\right\}, \\
& L_{k m} u_{j}=0, \quad j=1,2, \ldots, N .
\end{aligned}
$$

Consider operators $O_{j \lambda}$, acting in $L_{p}\left(G_{j} ; E\right)$ that is generated by boundary value problems (5.18). By virtue of Theorem 4.4 for all $f \in L_{p}\left(G_{j} ; E\right)$, for $\lambda \in S(\varphi)$, and sufficiently large $|\lambda|$ we have

$$
\sum_{k=1}^{n} \sum_{i=0}^{2}|\lambda|^{1-i / 2}\left\|D_{k}^{i} O_{j \lambda}^{-1} f\right\|_{p}+\left\|A O_{j \lambda}^{-1} f\right\|_{p} \leq C\|f\|_{p} .
$$

Extending $u_{j}$ zero on the outside of $\operatorname{supp} \varphi_{j}$ in equalities (5.27) and passing substitutions $u_{j}=O_{j \lambda}^{-1} v_{j}$ obtained from (5.1), operator equations with respect to $v_{j}$,

$$
v_{j}=K_{j \lambda} v_{j}+g_{j} f, \quad j=1,2, \ldots, N .
$$

By virtue of Theorem 2.3 and the estimate (5.28), in view of the smoothness of the coefficients of the expression $K_{j \lambda}$, and in view of condition (2.7) for $\lambda \in S(\varphi)$ and sufficiently large $|\lambda|$, we have $\left\|K_{j \lambda}\right\|<\varepsilon$, where $\varepsilon$ is sufficiently small. Consequently, (5.29) has unique solutions $v_{j}=\left[I-K_{j \lambda}\right]^{-1} g_{j} f$. Moreover,

$$
\left\|v_{j}\right\|_{p}=\left\|\left[I-K_{j \lambda}\right]^{-1} g_{j} f\right\|_{p} \leq\|f\|_{p} .
$$

Whence, $\left[I-K_{j \lambda}\right]^{-1} g_{j}$ are bounded linear operators from $L_{p}(G ; E)$ to $L_{p}\left(G_{j} ; E\right)$. Thus, we obtain that the functions

$$
u_{j}=U_{j \lambda} f=O_{j \lambda}^{-1-1}\left[I-K_{j \lambda}\right]^{-1} g_{j} f
$$


are solutions of (5.27). Consider a linear operator $(U+\lambda I)$ in $L_{p}(G ; E)$ such that

$$
(U+\lambda I) f=\sum_{j=1}^{N} \varphi_{j}(y) U_{j \lambda} f
$$

It is clear from the constructions $U_{j}$ and the estimate (5.28) that operators $U_{j \lambda}$ are bounded linear from $L_{p}(G ; E)$ to $W_{p}^{2}(G ; E(A), E)$ and for $\lambda \in S(\varphi)$ and sufficiently large $|\lambda|$ we have

$$
\sum_{k=1}^{n} \sum_{i=0}^{2}|\lambda|^{1-i / 2}\left\|D_{k}^{i} U_{j \lambda}^{-1} f\right\|_{p}+\left\|A U_{j \lambda}^{-1} f\right\|_{p} \leq C\|f\|_{p} .
$$

Therefore, $(U+\lambda I)$ is a bounded linear operator from $L_{p}$ to $L_{p}$. Then act of $O_{\lambda}$ to $u=$ $\sum_{j=1}^{N} \varphi_{j} U_{j \lambda} f$ gives $O_{\lambda} u=f+\sum_{j=1}^{N} \Phi_{j \lambda} f$, where $\Phi_{j \lambda}$ are linear combination of $U_{j \lambda}$ and $(d / d y) U_{j \lambda}$. By virtue of Theorem 2.3 and the estimate (5.33) from the expression $\Phi_{j \lambda}$ we obtain that operators $\Phi_{j \lambda}$ are bounded linear from $L_{p}(G ; E)$ to $L_{p}\left(G_{j} ; E\right)$ and $\left\|\Phi_{j \lambda}\right\|<\varepsilon$. Therefore, there exists a bounded inverse of operator $I+\sum_{j=1}^{N} \Phi_{j \lambda}$. Whence, we obtain that for all $f \in L_{p}(G ; E)$ the boundary value problem (3.1) has a unique solution

$$
u(x)=O_{\lambda}^{-1} f=\sum_{j=1}^{N} \varphi_{j} O_{j \lambda}^{-1}\left[I-K_{j \lambda}\right]^{-1} g_{j}\left(I+\sum_{j=1}^{N} \Phi_{j \lambda}\right)^{-1} f
$$

that is, we obtain assertion (a) of Theorem 5.3. Moreover, by virtue of (b), part of Theorem 5.2, operators $O_{j \lambda}$ are positive and operators

$$
\left[I-K_{j \lambda}\right]^{-1} g_{j}\left(I+\sum_{j=1}^{N} \Phi_{j \lambda}\right)^{-1}
$$

are bounded uniformly with respect $\lambda$. Then from (5.34) we obtain the assertion (b).

Result 5.4. Theorem 5.3 implies that the operator $O$ has a resolvent $(O+\lambda I)^{-1}$ for $\lambda \in$ $S(\varphi), \varphi \in(0, \pi / 3)$, and the following estimate holds:

$$
\sum_{k=1}^{n} \sum_{i=0}^{2}|\lambda|^{1-i / 2}\left\|D_{k}^{i}(O+\lambda I)^{-1}\right\|_{B\left(L_{p}(G ; E)\right)}+\left\|A(O+\lambda I)^{-1}\right\|_{B\left(L_{p}(G ; E)\right)} \leq C .
$$

Theorem 5.5. Let all conditions of Theorem 5.3 hold and $A^{-1} \in \sigma_{\infty}(E)$. Then the operator $O$ is Fredholm from $W_{p}^{2}(G ; E(A), E)$ into $L_{p}(G ; E)$.

Proof. Theorem 5.3 implies that the operator $O+\lambda I$ sufficiently large $|\lambda|$ have a bounded inverse $(O+\lambda I)^{-1}$ from $L_{p}(G ; E)$ to $W_{p}^{2}(G ; E(A), E)$, that is the operator $O+\lambda I$ is Fredholm from $W_{p}^{2}(G ; E(A), E)$ into $L_{p}(G ; E)$. Moreover, by virtue of Theorem 5.3 and the 
perturbation theory [12] we obtain that the operator $O$ is Fredholm from $W_{p}^{2}(G ; E(A), E)$ into $L_{p}(G ; E)$.

Remark 5.6. If $a_{k}$ are negative-valued functions, then (1.13), part of Condition 5.1, is satisfied for $0<\varphi \leq \pi$ and Theorems 5.2-5.5 are valid for $0<\varphi \leq \pi$.

Remark 5.7. Conditions $a_{k}\left(G_{j 0}\right)=a_{k}\left(G_{j b}\right), A\left(G_{k 0}\right)=A\left(G_{k b}\right)$ arise due to nonlocality of the boundary conditions (3.1). If boundary conditions are local then conditions mentioned above are not required any more.

5.2. IBVP for parabolic DOE. By applying Theorem 5.3 and by using [36, Theorem 4.2], we obtain the following.

Theorem 5.8. Let all conditions of Theorem 5.3 hold. Then the parabolic problem (3.2) for $\lambda \in S(\varphi), \varphi \in(0, \pi / 3)$ and sufficiently large $|\lambda|$ is maximal $L_{p}$-regular.

Proof. Really, problem (3.2) can be expressed in space $L_{p}\left(\mathbb{R}_{+} ; F\right)$ in the following form:

$$
\frac{d u}{d t}+O_{\lambda} u(t)=f(t), \quad u(0)=0, t>0
$$

where $F=L_{p}(G ; E)$ and $O$ is differential operator in $L_{p}(G ; E)$ generating by BVP (3.1). In view of (b), part of Theorem 5.3, operator $O$ is $\mathbb{R}$-positive in $L_{p}(G ; E)$ for $\varphi \in(0, \pi / 3)$. Then by virtue of [36, Theorem 4.2] we obtain the assertion.

Result 5.9. (a) If we put $a_{k}(x)=-1, A_{k}(x)=0, k=1,2, \ldots, n$, in (3.1) then, we obtain from Theorem 5.3 the maximal regularity, $\mathbb{R}$-positivity, and Fredholmness of Schrodinger type operator

$$
S_{1} u=-\Delta u(x)+A(x) u(x)
$$

with nonlocal boundary conditions in Banach-valued $L_{p}(G ; E)$ space.

(b) If we put $a_{k}(x)=-i, A_{k}(x)=0, k=1,2, \ldots, n$, in (3.2), then we obtain from Theorem 5.8 the maximal regularity of Schrodinger type operator

$$
S_{2} u=\frac{\partial u(x, t)}{\partial t}-i \Delta u(x, t)+A(x) u(x, t)
$$

with nonlocal boundary conditions in $L_{p}\left(G_{+} ; E\right)$ space.

5.3. Nonlocal boundary value problems for elliptic equations. The Fredholm property of boundary value problems for elliptic equations with parameters in smooth domains was studied in $[1-3,12,24]$ also for nonsmooth domains was investigated in $[17,21,28$, 34]. 
Let $\Omega \subset \mathbb{R}^{l}$ be an open connected set with compact $C^{2 m}$-boundary $\partial \Omega$. Let us consider a nonlocal boundary value problem on cylindrical domain $\widetilde{\Omega}=G \times \Omega$ for an elliptic differential equation

$$
\begin{gathered}
L u=\sum_{k=1}^{n} a_{k}(x) D_{k}^{2} u(x, y)+\sum_{k=1}^{n} d_{k}(x, y) D_{k} u(x, y) \\
+\sum_{|\alpha| \leq 2 m} a_{\alpha}(y) D_{y}^{\alpha} u(x, y)=f(x, y), \quad x \in G, y \in \Omega, \\
L_{k j} u=\alpha_{k j} D_{x}^{m_{k j}} u\left(G_{k 0}, y\right)+\beta_{k j} u D_{x}^{m_{k j}}\left(G_{k b}, y\right)=0, \quad j=1,2, k=1,2, \ldots, n, y \in \Omega, \\
B_{j} u=\sum_{|\beta| \leq m_{j}} b_{j \beta}(y) D_{y}^{\beta} u(x, y)=0, \quad x \in G, y \in \partial \Omega, j=1,2, \ldots, m,
\end{gathered}
$$

where $D_{j}=-i\left(\partial / \partial y_{j}\right), m_{k} \in\{0,1\}, \alpha_{k}, \beta_{k}, \delta_{k j i}$ are complex numbers, $y=\left(y_{1}, \ldots, y_{l}\right)$, and

$$
\begin{gathered}
G=\left\{x=\left(x_{1}, x_{2}, \ldots, x_{n}\right), 0<x_{k}<b_{k},\right\}, \\
G_{k 0}=\left(x_{1}, x_{2}, \ldots, x_{k-1}, 0, x_{k+1}, \ldots, x_{n}\right), \\
G_{k b}=\left(x_{1}, x_{2}, \ldots, x_{k-1}, b_{k}, x_{k+1}, \ldots, x_{n}\right), \\
m_{k} \in\{0,1\}, \quad D_{k}^{2}=\frac{\partial^{2}}{\partial x_{k}^{2}}, k=1,2, \ldots, n .
\end{gathered}
$$

Let $\Omega \subset \mathbb{R}^{l}$ be an open connected set with compact $C^{2 m}$-boundary $\partial \Omega$. Recall that for all $y_{0} \in \partial \Omega$ local coordinates corresponding to $y_{0}$ are defined as coordinates obtained from the original ones by a rotation and a shift which transfers $y_{0}$ to the origin and after which the positive $y_{l}$-axis has the direction of the interior normal to $\partial \Omega$ at $y_{0}$.

THeOREM 5.10. Let the following conditions be satisfied:

(1) $a_{\alpha} \in C(\bar{\Omega})$ for each $|\alpha|=2 m$, and $a_{\alpha} \in\left[L_{\infty}+L_{r_{k}}\right](\Omega)$ for each $|\alpha|=k<2 m$ with $r_{k} \geq q$ and $2 m-k>l / r_{k}$

(2) $b_{j \beta} \in C^{2 m-m_{j}}(\partial \Omega)$ for each $j, \beta, m_{j}<2 m$;

(3) for $y \in \bar{\Omega}, \xi \in \mathbb{R}^{l}, \lambda \in S(\varphi), \varphi \in(0, \pi / 3),|\xi|+|\lambda| \neq 0$, let $\lambda+\sum_{|\alpha|=2 m} a_{\alpha}(y) \xi^{\alpha} \neq 0$;

(4) for each $y_{0} \in \partial \Omega$ local $B V P$ in local coordinates corresponding to $y_{0}$

$$
\begin{gathered}
\lambda+\sum_{|\alpha|=2 m} a_{\alpha}\left(y_{0}\right) D^{\alpha} \vartheta(y)=0, \\
B_{j 0} \vartheta=\sum_{|\beta|=m_{j}} b_{j \beta}\left(y_{0}\right) D^{\beta} u(y)=h_{j}, \quad j=1,2, \ldots, m,
\end{gathered}
$$

has a unique solution $\vartheta \in C_{0}\left(\mathbb{R}_{+}\right)$for all $h=\left(h_{1}, h_{2}, \ldots, h_{m}\right) \in \mathbb{R}^{m}$, and for $\xi^{\prime} \in \mathbb{R}^{l-1}$ with

$$
\left|\xi^{\prime}\right|+|\lambda| \neq 0
$$


(5) $a_{k} \in C(\bar{G}), a_{k}(x) \neq 0$, and $a_{k} \neq b^{2}, b \in \mathbb{R}, x \in G, a_{k}\left(G_{j 0}\right)=a_{k}\left(G_{j b}\right), d_{k} \in L_{\infty}(G)$, $j=1,2, \ldots, n$;

Then

(6) $\eta_{k}=(-1)^{m_{k 1}} \alpha_{k 1} \beta_{k 2}-(-1)^{m_{k 2}} \alpha_{k 2} \beta_{k 1} \neq 0, k=1,2, \ldots, n$.

(a) the coercive estimate

$$
\begin{gathered}
\sum_{k=1}^{n}\left\|D_{k}^{2} u\right\|_{L_{p, q}(\tilde{\Omega})}+\sum_{|\beta|=2 m}\left\|D_{y}^{\beta} u\right\|_{L_{p, q}(\tilde{\Omega})}+\|u\|_{L_{p, q}(\tilde{\Omega})} \\
\leq C\left[\|L u\|_{L_{p, q}(\widetilde{\Omega})}+\|u\|_{L_{p, q}(\widetilde{\Omega})}\right]
\end{gathered}
$$

holds for the solution $u \in W_{p, q}^{2,2 m}(\widetilde{\Omega})$ of the problem (5.40);

(b) the problem (5.40) is Fredholm in $L_{p, q}(\widetilde{\Omega})$.

Proof. Let $E=L_{q}(G)$. Then by virtue of [18, Theorem 3.6] part (1) of Condition 5.1 is satisfied. Consider an operator $A$ which is defined by the equalities

$$
D(A)=W_{q}^{2 m}\left(G ; B_{j} u=0\right), \quad A u=\sum_{|\alpha| \leq 2 m} a_{\alpha}(y) D^{\alpha} u(y) .
$$

For $x \in G$ also consider operators

$$
A_{k}(x) u=d_{k}(x, y) u(y), \quad k=1,2, \ldots, n .
$$

The problem (5.40) can be rewritten in the form (3.1), where $u(x)=u(x, \cdot), f(x)=$ $f(x, \cdot)$ are functions with values in $E=L_{q}(G)$. By virtue of [12, Theorem 8.2] the problem

$$
\begin{gathered}
\lambda u(y)+\sum_{|\alpha| \leq 2 m} a_{\alpha}(y) D_{y}^{\alpha} u(y)=f(y), \\
B_{j} u=\sum_{|\beta| \leq m_{j}} b_{j \beta}(y) D_{y}^{\beta} u(y)=0, \quad j=1,2, \ldots, m,
\end{gathered}
$$

has a unique solution for $f \in L_{q}(G)$, and for $\arg \lambda \in S(\varphi),|\lambda| \rightarrow \infty$; and the differential operator $A$ generating by (5.47) is $\mathbb{R}$-positive in $L_{q}$. Then by virtue of (3.1)-(3.2) Condition 5.1 is fulfilled. It is known that the embedding $W_{q}^{2 m}(G) \subset L_{q}(G)$ is compact (see, e.g., [35, Theorem 3.2.5]). Then by using interpolation properties of Sobolev spaces (see, e.g., [35, Section 4]) it is clear to see that conditions (1.12) and (1.13) of Theorem 5.3 are fulfilled too.

5.4. Nonlocal boundary value problems for infinite systems of elliptic equations. Consider the following infinity systems of boundary value problem:

$$
\begin{gathered}
\sum_{k=1}^{n} a_{k}(x) D_{k}^{2} u_{m}(x)+\left(d_{m}(x)+\lambda\right) u_{m}(x)+\sum_{k=1}^{n} \sum_{j=1}^{\infty} d_{k m j}(x) D_{k} u_{j}(x) \\
=f_{m}(x), \quad x \in G, m=1,2, \ldots, \infty, \\
\alpha_{k j} D^{m_{k j}} u_{m}\left(G_{k 0}\right)+\beta_{k j} D^{m_{k j}} u_{m}\left(G_{k b}\right)=0, \quad j=1,2, k=1,2, \ldots, n,
\end{gathered}
$$


where

$$
\begin{gathered}
G=\left\{x=\left(x_{1}, x_{2}, \ldots, x_{n}\right), 0<x_{k}<b_{k},\right\}, \\
G_{k 0}=\left(x_{1}, x_{2}, \ldots, x_{k-1}, 0, x_{k+1}, \ldots, x_{n}\right), \\
G_{k b}=\left(x_{1}, x_{2}, \ldots, x_{k-1}, b_{k}, x_{k+1}, \ldots, x_{n}\right), \quad m_{k j} \in\{0,1\} .
\end{gathered}
$$

Let

$$
\begin{gathered}
D(x)=\left\{D_{m}(x)\right\}, \quad d_{m}>0, \quad u=\left\{u_{m}\right\}, \quad D u=\left\{d_{m} u_{m}\right\}, \quad m=1,2, \ldots, \infty, \\
l_{q}(D)=\left\{u: u \in l_{q},\|u\|_{l_{q}(D)}=\|D u\|_{l_{q}}=\left(\sum_{m=1}^{\infty}\left|d_{m} u_{m}\right|^{q}\right)^{1 / q}<\infty\right\}, \\
x \in G, \quad 1<q<\infty .
\end{gathered}
$$

Let $Q$ denote a differential operator in $L_{p}\left(G ; l_{q}\right)$ generating by problem $(5.48)$. Let

$$
B=B\left(L_{p}\left(G ; l_{q}\right)\right)
$$

Theorem 5.11. Let the following conditions hold:

(1) $a_{k} \in C(\bar{G}), a_{k}(x) \neq 0$, and $a_{k}(x) \neq 0$, and $a_{k} \neq b^{2}, b \in \mathbb{R}, x \in G, a_{k}\left(G_{j 0}\right)=a_{k}\left(G_{j b}\right)$, $j, k=1,2, \ldots, n$;

(2) $d_{m} \in C(G), d_{m \alpha} \in L_{\infty}(G)$ such that

$$
d_{m}\left(G_{j 0}\right)=d_{m}\left(G_{j b}\right), \quad \sum_{m, j=1}^{\infty} d_{m}^{q_{1}}(x) b_{k j m}^{-\left(q_{1} / 2\right)}<\infty, \frac{1}{q_{1}}+\frac{1}{q}=1,
$$

a.e. for $x \in G$ and $1<p, q<\infty$.

Then

(a) for all $f(x)=\left\{f_{m}(x)\right\}_{1}^{\infty} \in L_{p}\left(G ; l_{q}\right)$, for $\lambda \in S(\varphi), \varphi \in(0, \pi / 3)$, and for sufficiently large $|\lambda|$ the problem (5.48) has a unique solution $u=\left\{u_{m}(x)\right\}_{1}^{\infty}$ that belongs to space $W_{p}^{2}\left(G, l_{q}(D), l_{q}\right)$ and the coercive estimate

$$
\begin{gathered}
\sum_{k=1}^{n}\left[\left(\int_{G} \sum_{m=1}^{\infty}\left|D_{k}^{2} u_{m}(x)\right|^{q}\right)^{p / q} d x\right]^{1 / p}+\left[\left(\int_{G} \sum_{m=1}^{\infty}\left|d_{m} u_{m}(x)\right|^{q}\right)^{p / q} d x\right]^{1 / p} \\
\leq C\left[\left(\int_{G} \sum_{m=1}^{\infty}\left|f_{m}(x)\right|^{q}\right)^{p / q} d x\right]^{1 / p}
\end{gathered}
$$

holds for the solution of the problem (5.48); 
(b) for sufficiently large $|\lambda|>0$ there exists a resolvent $(Q+\lambda)^{-1}$ of operator $Q$ and

$$
\sum_{k=1}^{n} \sum_{j=0}^{2}(1+|\lambda|)^{1-j / 2}\left\|D_{k}^{j}(Q+\lambda)^{-1}\right\|_{B}+\left\|A(Q+\lambda)^{-1}\right\|_{B} \leq M
$$

(c) operator $Q$ is positive in $L_{p}\left(G ; l_{q}\right)$.

Proof. Really, let $E=l_{q}, A$, and $A_{k}(x)$ be infinite matrices, such that

$$
A=\left[d_{m} \delta_{m j}\right], \quad A_{\alpha}(x)=\left[d_{k j m}(x)\right], \quad m, j=1,2, \ldots, \infty .
$$

It is clear to see that this operator $A$ is positive in $l_{q}$. Therefore, by virtue of Theorem 5.3 we obtain that the problem (5.48) for all $f \in L_{p}\left(G ; l_{q}\right)$, for $\lambda \in S(\varphi), \varphi \in(0, \pi / 3)$, and sufficiently large $|\lambda|$ has a unique solution $u$ that belongs to space $W_{p}^{l}\left(G ; l_{q}(D), l_{q}\right)$ and

$$
\sum_{k=1}^{n}\left\|D_{k}^{2} u\right\|_{L_{p}\left(G ; l_{q}\right)}+\|d u\|_{L_{p}\left(G ; l_{q}\right)} \leq C\|f\|_{L_{p}\left(G ; l_{q}\right)} .
$$

From the above estimate we obtain (5.53). The estimate (5.54) is obtained from Result 5.4.

5.5. IBVP for infinite systems of parabolic equations. Consider an infinity system of parabolic nonlocal IBVP:

$$
\begin{gathered}
\frac{\partial u_{m}(t, x)}{\partial t}+\sum_{k=1}^{n} a_{k}(x) D_{k}^{2} u_{m}(t, x)+\left(d_{m}(x)+\lambda\right) u_{m}(t, x) \\
+\sum_{k=1}^{n} \sum_{j=1}^{\infty} d_{k m j}(x) D_{k} u_{j}(t, x)=f_{m}(t, x), \quad m=1,2, \ldots, \infty, \\
L_{k j} u_{m}=\alpha_{k j} D^{m_{k j}} u_{m}\left(G_{k 0}\right)+\beta_{k j} D^{m_{k j}} u_{m}\left(G_{k b}\right)=0, \\
j=1,2, \quad k=1,2, \ldots, n, u_{m}(0, x)=0, t \in \mathbb{R}_{+}, x \in G .
\end{gathered}
$$

Theorem 5.12. Let all conditions of Theorem 5.11 hold. Then the parabolic systems (5.57) for $\lambda \in S(\varphi), \varphi \in(0, \pi / 3)$, and for sufficiently large $|\lambda|$ have the property of maximal $L_{p^{-}}$ regularity.

Proof. Really, let $E=l_{q}, A$, and $A_{k}(x)$ be infinite matrices, such that

$$
A=\left[d_{m} \delta_{m j}\right], \quad A_{\alpha}(x)=\left[d_{k j m}(x)\right], \quad m, j=1,2, \ldots, \infty .
$$


Then the problem (5.57) can be expressed in the form

$$
\begin{gathered}
\frac{\partial u(t, x)}{\partial t}+\sum_{k=1}^{n} a_{k}(x) D_{k}^{2} u(t, x)+A_{\lambda} u(t, x)+\sum_{k=1}^{n} A_{k}(x) \frac{\partial u(t, x)}{\partial x_{k}}=f(t, x), \\
L_{k j} u=\left[\alpha_{k j} u^{\left(m_{k j}\right)}\left(t, G_{k 0}\right)+\beta_{k j} u^{\left(m_{k j}\right)}\left(t, G_{k b}\right)\right]=0, \\
u(0, x)=0, \quad j=1,2, k=1,2, \ldots, n, t \in \mathbb{R}_{+}, x \in G \subset \mathbb{R}^{n},
\end{gathered}
$$

where

$$
A=\left[d_{m} \delta_{m j}\right], \quad A_{\alpha}(x)=\left[d_{k j m}(x)\right], \quad m, j=1,2, \ldots, \infty .
$$

Then by virtue of Theorems 5.3 and 5.8 we obtain the assertion.

Remark 5.13. There are many positive operators in the different concrete Banach spaces. Therefore, putting concrete Banach spaces instead of $E$ and concrete positive differential, pseudodifferential operators or finite or infinite matrices for instance, instead of operator $A$, on DOE's (3.1) or (3.2) by virtue of Theorems 5.3, 5.8 we can obtain different class of maximal regular and Fredholm boundary value problems for partial differential or pseudodifferential equations, and maximal regular parabolic problems or their finite and infinite systems.

\section{Acknowledgment}

This work was supported by project no UDP-543/17062005 of Istanbul University.

\section{References}

[1] S. Agmon, On the eigenfunctions and on the eigenvalues of general elliptic boundary value problems, Communications on Pure and Applied Mathematics 15 (1962), 119-147.

[2] S. Agmon and L. Nirenberg, Properties of solutions of ordinary differential equations in Banach space, Communications on Pure and Applied Mathematics 16 (1963), 121-239.

[3] M. S. Agranovič and M. I. Višik, Elliptic problems with a parameter and parabolic problems of general type, Uspekhi Matematicheskikh Nauk 19 (1964), no. 3 (117), 53-161.

[4] A. Aibeche, Coerciveness estimates for a class of nonlocal elliptic problems, Differential Equations and Dynamical Systems 1 (1993), no. 4, 341-351.

[5] H. Amann, Linear and Quasilinear Parabolic Problems. Vol. I, Monographs in Mathematics, vol. 89, Birkhäuser Boston, Massachusetts, 1995.

[6] A. Ashyralyev, On well-posedness of the nonlocal boundary value problems for elliptic equations, Numerical Functional Analysis and Optimization 24 (2003), no. 1-2, 1-15.

[7] J.-P. Aubin, Abstract boundary-value operators and their adjoints, Rendiconti del Seminario Matematico della Università di Padova 43 (1970), 1-33.

[8] O. V. Besov, V. P. Il'in, and S. M. Nikol'skiü, Integral Representations of Functions, and Embedding Theorems, Izdat. "Nauka", Moscow, 1975.

[9] J. Bourgain, Vector-valued singular integrals and the $H^{1}-B M O$ duality, Probability Theory and Harmonic Analysis (Cleveland, Ohio, 1983), Monogr. Textbooks Pure Appl. Math., vol. 98, Dekker, New York, 1986, pp. 1-19.

[10] D. L. Burkholder, A geometric condition that implies the existence of certain singular integrals of Banach-space-valued functions, Proceedings of Conference on Harmonic Analysis in Honor of 
Antoni Zygmund, Vol. I, II (Chicago, Ill, 1981), Wadsworth Math. Ser., Wadsworth, California, 1983, pp. 270-286.

[11] Ph. Clément, B. de Pagter, F. A. Sukochev, and H. Witvliet, Schauder decomposition and multiplier theorems, Studia Mathematica 138 (2000), no. 2, 135-163.

[12] R. Denk, M. Hieber, and J. Prüss, $\mathscr{R}$-boundedness, Fourier multipliers and problems of elliptic and parabolic type, Memoirs of the American Mathematical Society 166 (2003), no. 788, viii+114.

[13] J. Diestel, H. Jarchow, and A. Tonge, Absolutely Summing Operators, Cambridge Studies in Advanced Mathematics, vol. 43, Cambridge University Press, Cambridge, 1995.

[14] G. Dore and A. Venni, On the closedness of the sum of two closed operators, Mathematische Zeitschrift 196 (1987), no. 2, 189-201.

[15] G. Dore and S. Yakubov, Semigroup estimates and noncoercive boundary value problems, Semigroup Forum 60 (2000), no. 1, 93-121.

[16] V. I. Gorbachuk and M. L. Gorbachuk, Boundary Value Problems for Operator-Differential Equations, "Naukova Dumka", Kiev, 1984.

[17] P. Grisvard, Elliptic Problems in Nonsmooth Domains, Monographs and Studies in Mathematics, vol. 24, Pitman, Massachusetts, 1985.

[18] R. Haller, H. Heck, and A. Noll, Mikhlin's theorem for operator-valued Fourier multipliers in n variables, Mathematische Nachrichten 244 (2002), no. 1, 110-130.

[19] M. Hieber and J. Prüss, Heat kernels and maximal $L^{p}-L^{q}$ estimates for parabolic evolution equations, Communications in Partial Differential Equations 22 (1997), no. 9-10, 1647-1669.

[20] T. Kato, Perturbation Theory for Linear Operators, Izdat. “Mir”, Moscow, 1972.

[21] V. A. Kondratiev and O. A. Oleinik, Boundary value problems for partial differential equations in non-smooth domains, Russian Mathematical Surveys 38 (1983), no. 2, 1-86.

[22] S. G. Kreĭn, Linear Differential Equations in Banach Space, American Mathematical Society, Rhode Island, 1971.

[23] D. Lamberton, Équations d'évolution linéaires associées à des semi-groupes de contractions dans les espaces $L^{p}$, Journal of Functional Analysis 72 (1987), no. 2, 252-262.

[24] J.-L. Lions and E. Magenes, Problems and limites non homogenes, Journal d'Analyse Mathématique 11 (1963), 165-188.

[25] J.-L. Lions and J. Peetre, Sur une classe d'espaces d'interpolation, Institut des Hautes Études Scientifiques. Publications Mathématiques (1964), no. 19, 5-68.

[26] P. I. Lizorkin, $\left(L_{p}, L_{q}\right)$-multipliers of Fourier integrals, Doklady Akademii Nauk SSSR 152 (1963), no. $4,808-811$.

[27] T. R. McConnell, On Fourier multiplier transformations of Banach-valued functions, Transactions of the American Mathematical Society 285 (1984), no. 2, 739-757.

[28] S. A. Nazarov and B. A. Plamenevsky, Elliptic Problems in Domains with Piecewise Smooth Boundaries, de Gruyter Expositions in Mathematics, vol. 13, Walter de Gruyter, Berlin, 1994.

[29] V. B. Shakhmurov, The compactness of an imbedding into nonisotropic spaces of vector-valued functions and applications, Doklady Akademii Nauk SSSR 241 (1978), no. 6, 1285-1287.

[30] _ Theorems on imbedding compactness in weighted anisotropic spaces and their applications, Doklady Akademii Nauk SSSR 291 (1986), no. 6, 1305-1309.

[31] Embedding theorems and their applications to degenerate equations, Differential Equations 24 (1988), no. 4, 475-482.

[32] __ Coercive boundary value problems for regular degenerate differential-operator equations, Journal of Mathematical Analysis and Applications 292 (2004), no. 2, 605-620.

[33] __ Embedding operators and maximal regular differential-operator equations in Banachvalued function spaces, Journal of Inequalities and Applications 2005 (2005), no. 4, 329-345.

[34] A. L. Skubachevskii, Elliptic Functional-Differential Equations and Applications, Operator Theory: Advances and Applications, vol. 91, Birkhäuser, Basel, 1997. 


\section{Maximal regular problems}

[35] H. Triebel, Interpolation Theory, Function Spaces, Differential Operators, North-Holland Mathematical Library, vol. 18, North-Holland, Amsterdam, 1978.

[36] L. Weis, Operator-valued Fourier multiplier theorems and maximal $L_{p}$-regularity, Mathematische Annalen 319 (2001), no. 4, 735-758.

[37] S. Yakubov, Completeness of Root Functions of Regular Differential Operators, Pitman Monographs and Surveys in Pure and Applied Mathematics, vol. 71, Longman Scientific \& Technical, Harlow, 1994.

[38] _ A nonlocal boundary value problem for elliptic differential-operator equations and applications, Integral Equations and Operator Theory 35 (1999), no. 4, 485-506.

[39] S. Yakubov and Y. Yakubov, Differential-Operator Equations. Ordinary and Partial Differential Equations, Chapman \& Hall/CRC Monographs and Surveys in Pure and Applied Mathematics, vol. 103, Chapman \& Hall/CRC, Florida, 2000.

Veli B. Shakhmurov: Department of Electrical-Electronics Engineering, Faculty of Engineering, Istanbul University, Avcilar 34320, Istanbul, Turkey

E-mail address: sahmurov@istanbul.edu.tr 


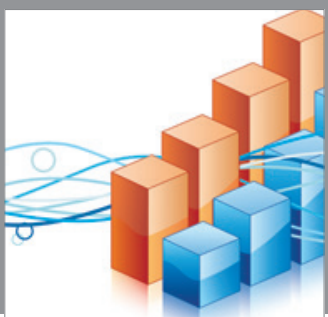

Advances in

Operations Research

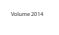

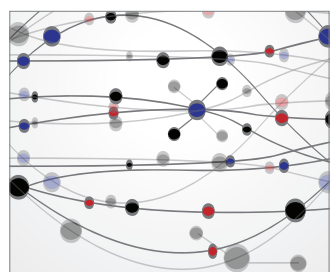

\section{The Scientific} World Journal
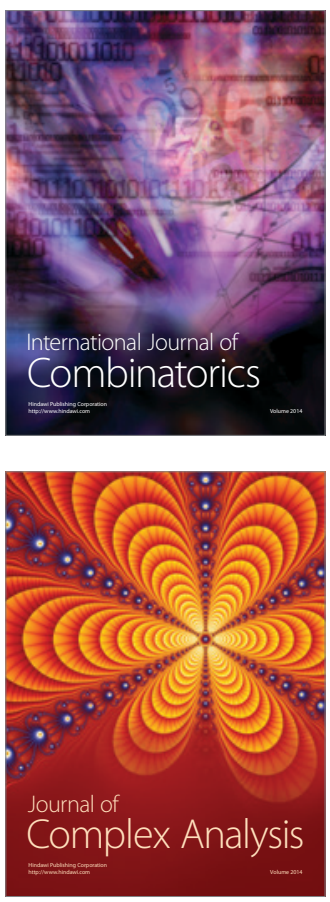

International Journal of

Mathematics and

Mathematical

Sciences
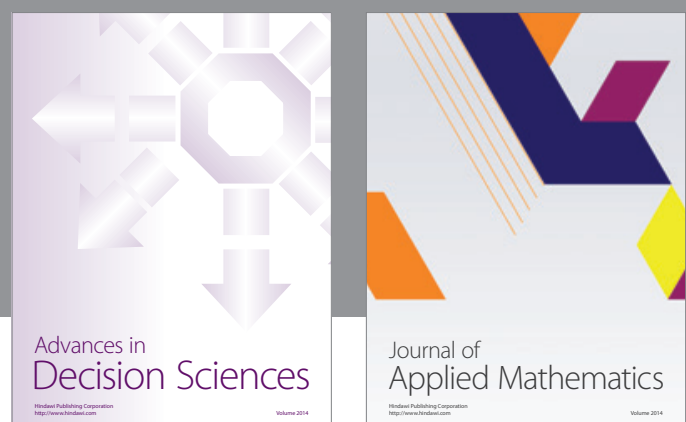

Journal of

Applied Mathematics
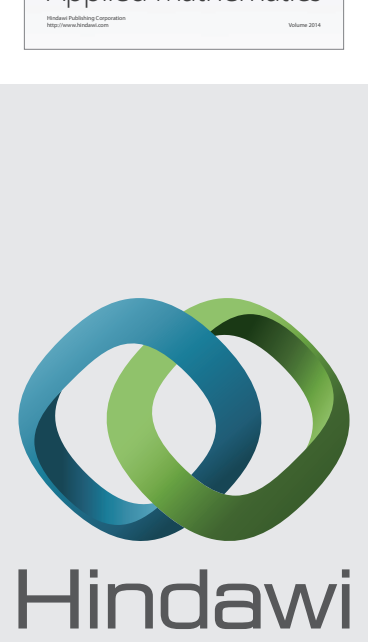

Submit your manuscripts at http://www.hindawi.com
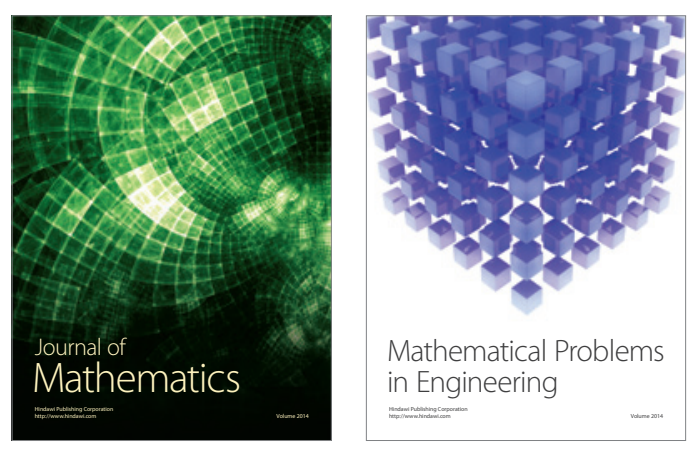

Mathematical Problems in Engineering
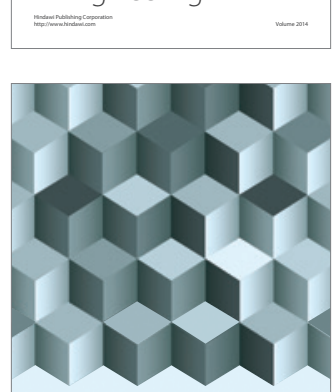

Journal of

Function Spaces
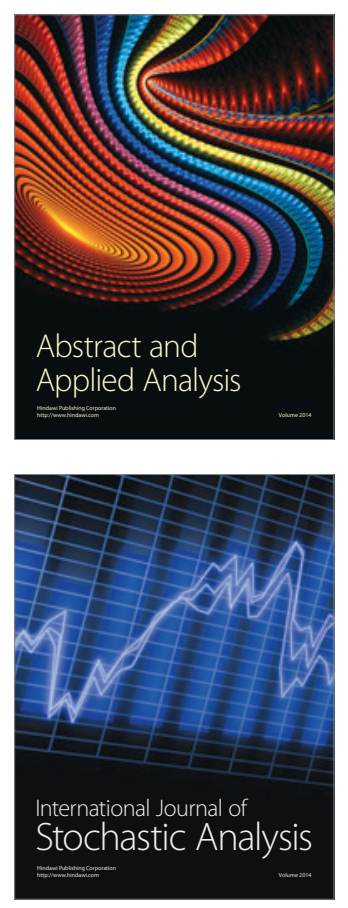

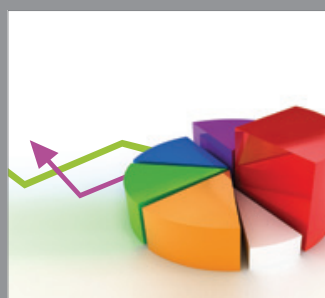

ournal of

Probability and Statistics

Promensencen
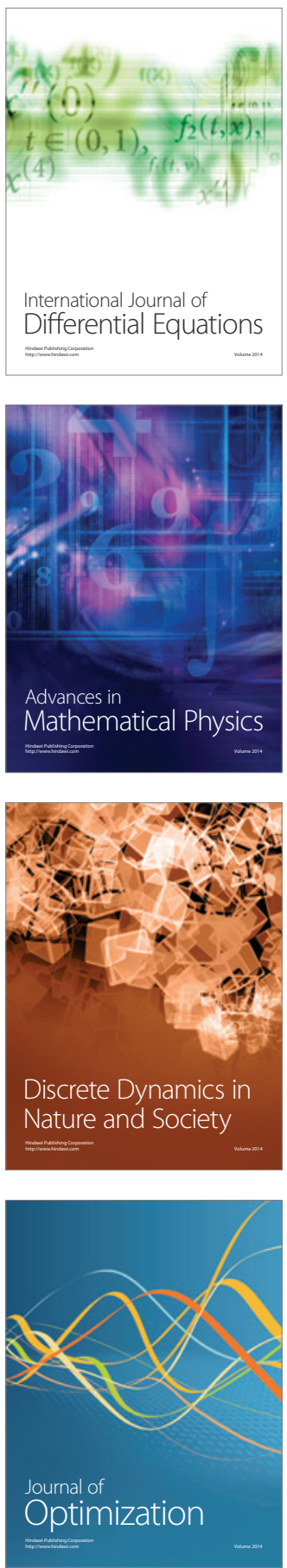